\title{
LA DIMENSIÓN ESPACIAL DEL SUICIDIO Y SU VÍNCULO CON EL MERCADO LABORAL MEXICANO (2000-2004)*
}

\author{
ROSALBA JASSO VARGAS" \\ EL COLEGIO DE MÉXICO
}

Recibido/Received/Recebido: 21/11/2012 - Aceptado/ Accepted / Aprovado: 15/04/2013

\begin{abstract}
Resumen
A principios del siglo XXI en México se ha puesto de manifiesto en la opinión pública la preocupación respecto al ascenso de muertes por la causa de suicidio y de su posible vínculo con malas condiciones laborales. El objetivo principal del trabajo es comprobar si las tasas de suicidio son una respuesta a malas condiciones laborales. Para este propósito, partiendo de una perspectiva espacial, se buscó evidenciar el comportamiento heterogéneo del fenómeno del suicidio a través del territorio mexicano, verificar la existencia de "regiones" en el espacio de tasas de suicidio y comprobar si las tasas de suicidio son una respuesta a las características económicas, demográficas y sociales de los municipios de la República Mexicana en particular a las laborales. El trabajo encuentra que no existen las evidencias estadísticas suficientes para probar que malas condiciones laborales estén provocando un incremento en la incidencia del suicidio. Adicionalmente, se hace visible que las cifras nacionales y estatales no reconocen comportamientos regionales importantes. Palabras clave: Suicidio, Análisis espacial, Condiciones laborales, Clústeres, Modelo rezago.

\section{THE SPATIAL DIMENSION OF SUICIDE AND ITS LINK TO THE MEXICAN LABOR MARKET (2000-2004)}

\begin{abstract}
At the beginning of XXI century in Mexico it has been revealed the population's concern of death increase related to suicides and its possible link to poor working conditions. The main objective of this study is to corroborate if suicide rate is an answer to poor working conditions. For this propose, from a spatial perspective, we sought evidence of the heterogeneous behavior of suicide phenomenon through Mexican territory and verify the existence of "regions" in the suicide rate and check if suicide rates are a response to the economic, demographic and social municipalities of Mexico Republic specially to labor conditions. The study finds that there is not enough statistical evidence to prove that poor working conditions are causing an increase in suicide incidence. Additionally, it is noted that national and state figures do not recognize important regional behaviors.

Keywords: Suicide, Spatial Analysis, Working conditions, Clustering, Lag model.
\end{abstract}

Resultados del proyecto de investigación del mismo nombre desarrollado en el Colegio de México.

Actuaria de la Universidad Nacional Autónoma de México y maestra en Demografía del Colegio de México. Correo electrónico: rjasso@colmex.mx 


\title{
A DIMENSÃO ESPACIAL DO SUICÍDIO E SEU VÍNCULO COM O MERCADO TRABALHISTA MEXICANO (2000-2004)
}

\section{Resumo}

\begin{abstract}
Nos princípios do século XXI no México a opinião pública manifestou sua preocupação com o aumento de suicídios e seu possível vínculo com as más condições de trabalho. O objetivo principal deste texto é comprovar se as taxas de suicídio são uma resposta às más condições de trabalho. Para este propósito, partindo de uma perspectiva espacial, procurou-se evidenciar o comportamento heterogêneo do fenômeno do suicídio através do território mexicano, verificar a existência de "regiões" no espaço de taxas de suicídio e comprovar se as taxas de suicídio são uma resposta às características econômicas, demográficas e sociais dos municípios da República Mexicana em particular às situações de trabalho. O texto encontra que não existem as evidências estatísticas suficientes para provar que as más condições de trabalho estejam provocando um incremento na incidência do suicídio. Adicionalmente, torna-se visível que as cifras nacionais e estatais não reconhecem comportamentos regionais importantes.
\end{abstract}

Palavras chave: Suicídio, Análise espacial, Condições de trabalho, Clusters, Modelo resíduo.

Vargas, J. (2013) La dimensión espacial del suicidio y su vínculo con el mercado laboral mexicano (2000-2004). En: Revista de la Facultad de Ciencias Económicas de la Universidad Militar Nueva Granada. rev.fac.cienc.econ, XXI (1).

JEL: J01, J81, R11, L29.

\section{Introducción}

Durkheim (1897) define al suicidio como: "todo caso de muerte que resulte, directa o indirectamente, de un acto, positivo o negativo, realizado por la víctima misma, sabiendo ella que debía producir este resultado". Según Durkheim el materialismo económico vulnera al hombre a un eterno descontento por disponer de aspiraciones más altas donde al no lograrlas no tiene las fuerzas para soportarlo. En este trabajo consideramos que el suicidio es el acto de quitarse la vida sin importar los motivos que impulsaron al individuo a tomar tal decisión ${ }^{1}$.

Guilherme Borges con otros investigadores publicaron en 1996 que la tasa de suicidio en México había aumentado en 156\% en el periodo de 1970 a 1994 y advirtieron que: "El suicidio era un pro- blema ascendente en México que afectaba, en forma principal, a los hombres de edad avanzada. Sin embargo, al observar estos incrementos en la población adolescente y adulta joven se enfatizaba la necesidad de desarrollar programas de prevención y tratamiento en esta área" (Universal, 24 de noviembre 2009). De hecho el fenómeno del suicidio es un tema de suma relevancia y hoy es considerado como un problema de salud pública en el mundo. Por ello, la OMS (2010) establece que "El suicidio constituye un problema de salud pública muy importante pero en gran medida prevenible, que provoca casi la mitad de todas las muertes violentas y se traduce en casi un millón de víctimas al año, además de unos costos económicos cifrados en miles de millones de dólares".

Por su parte, el suicidio en México es un fenómeno que hacia finales del siglo pasado ya cobraba en

1 El suicidio, como es definido por el Instituto Nacional de Estadística y Geografía (INEGI) en la Síntesis Metodológica de las Estadísticas de Intentos de Suicidios y Suicidios, se refiere a las personas cuyo acto o conducta destruye su propia existencia (INEGI, 2003). 
promedio 3467 vidas al año², 2909 hombres y 558 mujeres. Las notas periodísticas argumentaron que los nuevos detonadores del suicidio en México se debían al desempleo, al trabajo "precario", al hecho de no tener prestaciones ni estabilidad económica y contar con redes familiares y sociales escasas. Además, las cifras en México respecto al número de suicidios juveniles se dispararon entre 1970 y 2007, siendo el suicidio la quinta causa de muerte para menores de 15 años y la tercera para jóvenes entre 15 y 24 años (Milenio, 10 de septiembre 2009). En esta misma nota se menciona que los jóvenes mexicanos ven un oscuro futuro ante las condiciones actuales pues no saben en dónde hallarán empleo o cómo podrán enfrentar las dificultades cotidianas.

Los resultados en los diferentes estudios en México asocian un mayor riesgo de suicidio a grupos de edad extremos: en 1979-1993, los grupos fueron 20-24 años y 70 años y más (Hijar, Rascon, Blanco \& Lopez, 1996); en 1996-2001, en el caso de los hombres fueron los grupos de 20-34 años y 60 años y para las mujeres los grupos de 11-19 años y 20-39 años de edad para el caso de las mujeres (Puentes, López \& Martínez, 2004). En los últimos 50 años, el mayor riesgo de suicidio para los hombres lo enfrenta el grupo de los 75 años o más y de 15-24 años para el caso de las mujeres (Hernández \& Flores, 2011).

Las entidades que presentaban una mayor incidencia de suicidios eran las entidades de Tabasco, Campeche, Yucatán, Quintana Roo, Baja California Sur, Sonora, Chihuahua y Jalisco. Esta distribución espacial del fenómeno despertó la curiosidad en torno a dos preguntas, por qué el fenómeno del suicidio ocurre de manera diferenciada en el país y qué factores pueden explicar o estar relacionados con dicha distribución. Regiones de tasas altas (bajas) de suicidio, llamados clústeres, ${ }^{3}$ pueden responder a características del municipio o características com- partidas con unidades vecinas. En el presente trabajo se busca poner a prueba la hipótesis de que, en el caso de México, son las características del municipio y las condiciones laborales las que explican este agrupamiento de tasas. ${ }^{4}$

A pesar de su importancia, los estudios del suicidio en México principalmente nos han proporcionado información sobre las características sociodemográficas de la población suicida y las entidades de mayor incidencia, es decir, los estudios realizados en nuestro país fundamentalmente han observado el evento desde la perspectiva del individuo. El objetivo aquí es observar al suicidio en su nivel agregado a una escala municipal.

En años más recientes Hernández \& Flores (2011: 82-83) en su documento "El suicidio en México" mencionan como el fenómeno del suicidio ha presentado una dinámica en ascenso sobre todo entre los jóvenes en edades activas y hombres mayores de edad. Estos autores comparan las tendencias en las tasas de suicidio con otras muertes violentas como el homicidio y accidentes mostrando que mientras las primeras tienen un comportamiento creciente, las últimas tienen un descenso continuo hasta $2006 .^{5}$

Aunque este documento se limitará al fenómeno del suicidio en el periodo 2000-2004 consideramos relevante percatarnos de la tendencia del fenómeno en años cercanos. En la Ilustración 1, la población masculina muestra una tendencia al alza de las tasas $^{6}$ de suicidio con ligeras caídas en los años 19981999, 2003, 2006-2007, 2010 y nuevamente hay un considerable aumento en la tasa para el año 2011. No descartamos que las pequeñas caídas en esta tendencia puedan explicarse por un problema de cobertura. En el caso de las mujeres también presenta una tendencia al alza que no supera los 20 suicidios por millón de habitantes. Ante esta tendencia a la alza confirmamos la relevancia de obtener análi-

\footnotetext{
Promedio de cuatro años (1998 a 2001), cifras tomadas de las defunciones de Estadísticas Vitales proporcionadas por INEGI.

Valores similares en unidades geográficamente cercanas.

En el entendido de que dichas características también se agrupan en el espacio.

Por el repunte probable que se tuvo en este año por la situación coyuntural de la lucha contra el narcotráfico.

Tasas estandarizadas por el método indirecto utilizando como estándar la población de México en el año 2010.
} 
sis más detallados respecto a las posibles relaciones entre el fenómeno y las características económicas, demográficas y sociales de los municipios en el país. La importancia del tema radica no únicamente en el número de vidas que cobra sino que la incidencia del suicidio pudiera ser un reflejo de las malas condiciones de vida en que se vive en algunas regiones del país, en particular las laborales.

La razón principal por la que tomamos este periodo "2000-2004" es el hecho de que la opinión pública en este periodo relacionó el incremento de la incidencia del suicidio con problemas del mercado laboral mientras que en periodos recientes se vinculó con otros factores ${ }^{7}$ ya no exclusivos de lo laboral o económico. A pesar de que el fenómeno del suicidio presenta un incremento en sus tasas para años recientes, la preocupación más bien se ha volcado al incremento en las tasas de homicidio después del 2007 probable resultado de la guerra contra el crimen organizado implementada por el gobierno mexicano. La segunda razón es que dado que el registro de la causa de muerte como presunto suicidio está sujeta a investigación legal por parte del ministerio Público y algunos casos de homicidios pudieran ser catalogados como suicidios, pretendemos analizar un periodo un tanto más estable en cuanto a las cifras catalogadas como muertes violentas y no como la de años recientes que pudiera verse afectada por el incremento de los homicidios. Otro motivo tiene que ver con los montos poblacionales necesarios para el cálculo de las tasas a una escala municipal. El Consejo Nacional de Población ya nos ha presentado las cifras conciliadas a una escala estatal de acuerdo a la información proporcionada por el XII Censo de Población y Vivienda 2010, sin embargo, los demógrafos aún se encuentran discutiendo el comportamiento de la dinámica demográfica en estos años cercanos al Censo 2010.

Pese al incremento continuo, las tasas de suicidio en México son bajas respecto a otros países (Ilustración 2) ${ }^{8}$. México ocupa en 2008 el lugar 147 de 193 paí- ses. Tal vez como consecuencia de esto, estudios en otros países muestran una mayor preocupación respecto a la relación de suicidio-desempleo (o suicidio con condiciones laborales). En el caso de México no tenemos un estudio que ahonde demasiado en la relación suicidio-condiciones económicas y el fenómeno únicamente se aborda de manera descriptiva. En América Latina las tasas de suicidio no superan los 13 por 100 mil habitantes. Las tasas más altas corresponden a Nicaragua, Cuba y Chile (Hernández \& Flores, 2011, 74). México no ha superado los 5 suicidios por 100 mil habitantes si se analiza la evolución de sus tasas de 1950 a 2008 (Hernández \& Flores, 2011, 76).

Ilustración 1. Evolución de las tasas estandarizadas de suicidio en México (190-2011)

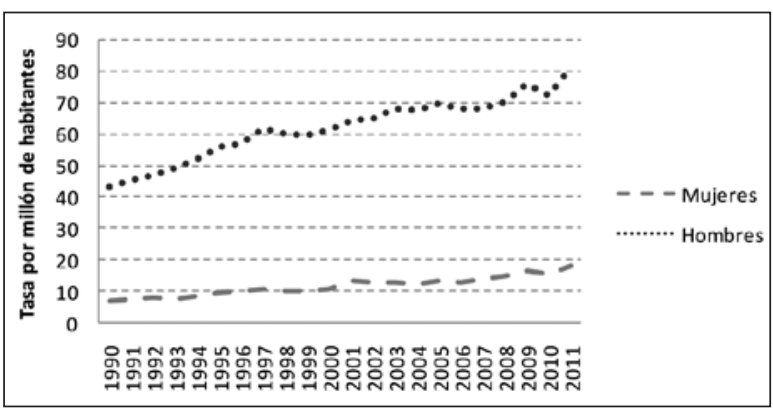

Ilustración 2. Tasas de suicidio estandarizadas por millón de habitantes, $2008^{10}$

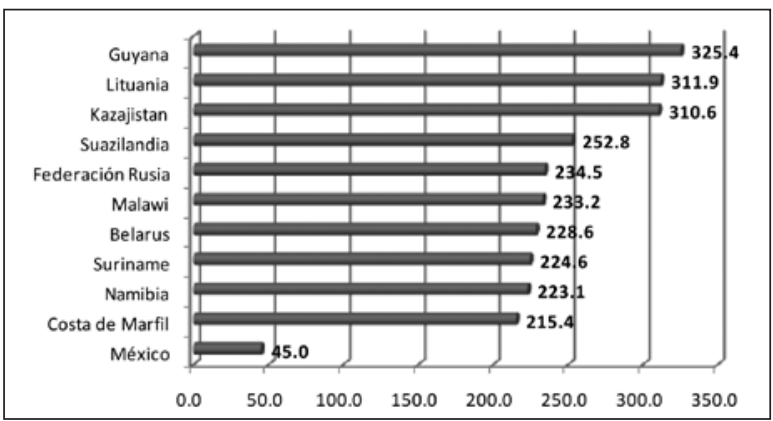

7 Consumo del alcohol o drogas (Jornada, 3 de enero 2013), el desamor y los problemas familiares y económicos (Centro Universitario de Ciencias de la Salud, Septiembre 2012), o bien, con los trastornos mentales mal atendidos (Jornada, 3 de abril 2013), la desesperanza, la falta de redes de apoyo (Educación a Debate, 2 de enero 2013).

8 La información de suicidios se basan en suicidios consumados aunque las cifras pudieran ser incorrectas debido a un problema de subregistro. A nivel mundial hay consenso de que el número de suicidios se encuentra subregistrado (Hijar et al., 1996).

9 Fuente: INEGI, Estadísticas Vitales 1990-2011. CONAPO, Proyecciones de Población basadas en el XII Censo de Población 2010

10 Fuente: OMS, World Health Report 2010. 
En la tarea que nos hemos encomendado, suponemos que el espacio juega un papel determinante en la ocurrencia del suicidio en nuestro país, sin embargo, no existen estudios donde se haga uso de las técnicas de análisis de datos geo-referenciados que permitan explicar la posible regionalización del fenómeno del suicidio en México y dar cuenta sobre la existencia o no de un proceso de difusión (o mejor dicho a condiciones económicas y sociales parecidas en contextos cercanos que dan como resultado agrupamientos de las tasas de suicidio). Es importante identificar el lugar donde ocurren los suicidios pues permite indagar respecto a la población vulnerable de acuerdo con algunas características del municipio y, en particular, la referente a la calidad del empleo de la región. Así, este documento trata de provocar el interés de incorporar la variable espacial cuando se analiza el fenómeno del suicidio tanto en México como en América Latina.

Hemos considerado la relación suicidio-condiciones laborales pues una de las dimensiones más importantes de los aspectos sociales es la vinculada con el empleo, dado que la mayor parte de las personas viven de su trabajo y esto incide en muchos aspectos de su vida construyendo identidades laborales o no laborales y en ese sentido es una esfera importante de la integración social.

Ahora bien, ¿cuáles son las condiciones laborales en México a inicios del siglo XXI? Brígida García (1999) resume de manera muy clara los principales problemas laborales que enfrentó México a principios del siglo XXI. La autora menciona que México se encontraba lejos de poder ofrecer empleos satisfactorios a todos los que los demandan. Recordemos que México atraviesa por la etapa de la transición demográfica que algunos han llamado como "bono demográfico" que representa un cambio en las tasas de dependencia siendo en este momento más bajas que lo que fueron en el pasado (debido a la dependencia infantil) y de lo que serán en el futuro (dependencia de adultos mayores), sin embargo en nuestro caso la preocupación no radica en el cambio de las tasas de dependencia sino en el reto que representan los volúmenes poblacionales que demandan empleos de calidad.
García menciona que el principal problema en la cuestión laboral era la insuficiencia de empleos que permitieran la sobrevivencia digna. Respecto a la magnitud de este problema, menciona algunos indicadores que permiten vislumbrar la situación del país a finales del siglo XX. El primer dato consiste en el aumento en trabajadores por cuenta propia $y$ familiares no remunerados que representaron en los noventas $37 \%$ de la fuerza de trabajo, cifra que habla del carácter excluyente de la estrategia de desarrollo capitalista que ha seguido el país. Adicionalmente, se tiene que entre los cuenta propia y no remunerados se concentra gran parte de los pobres de México (en 1997 38\% de los trabajadores cuenta propia recibía menos de un salario mínimo). El siguiente dato relevante es el crecimiento de los micronegocios, que indica la polarización creciente del mercado de trabajo, establecimientos que en su mayoría cuentan con escaso capital y tecnología y poco acceso al crédito y su mercado de productos es restringido y fluctuante. En general, las condiciones de trabajo eran muy difíciles para la fuerza de trabajo en conjunto y esto está apoyado con el hecho de que 65\% de la PEA en 1997 recibía como máximo dos salarios mínimos o no recibía ingresos, $57 \%$ de los asalariados no contaba con ninguna forma de prestación social y 46\% sólo tenía un contrato de trabajo verbal.

La población ocupada, en México, sin seguridad social a nivel nacional desde 1991 al 2006 se mantuvo alrededor del $60 \%$ y no sólo no recibían seguridad social sino ninguna prestación (Pacheco, 2009). Pacheco \& Flores (2005) ponen en evidencia que las condiciones laborales del conjunto de trabajadores eran deficientes y que los trabajadores asalariados que en otro tiempo eran el modelo a seguir en ese momento tendieron a comportarse como los grupos que se consideran marginales o excluidos. Los grupos más afectados eran los jóvenes por la dificultad de inserción a un primer trabajo y por la inestabilidad en los empleos a que tenían acceso. La mayoría de los empleos que se generaron para los jóvenes se crearon en el sector informal.

El siguiente apartado presenta una revisión de los hallazgos encontrados en torno al fenómeno del suicidio y las relaciones que se establecen con las condiciones económicas. Se presenta además los 
argumentos que hacen referencia a la perspectiva espacial, una breve reflexión sobre las variables a considerar del mercado laboral y nuestro interés de abordar el eje de análisis de trabajo decente. Después se describe brevemente las fuentes de información disponibles y la metodología utilizada. Se agrega un apartado en el cual se describen los pasos que se siguieron y los instrumentos empleados dentro del análisis espacial. Y finalmente se presentan los resultados del modelo de regresión espacial y los hallazgos en cuanto a la relación suicidio-trabajo y se destina un apartado a modo de conclusión.

\section{Hallazgos relevantes para la discusión}

Es importante mencionar que el número de trabajos del tipo epidemiológico es amplio y se pueden mencionar al menos 20 (Gómez \& Borges, 1996). La epidemiologia descriptiva puede resumir que el perfil del suicida en México es de sexo predominante masculino; de menos de 40 años; no ocurre más en ningún mes del año; las sustancias tóxicas, las armas de fuego o los medios para ahorcarse están a su disposición en su entorno; su estado civil puede ser soltero o casado y su ocupación es de empleado. También existen esfuerzos de estudios del suicidio desde una perspectiva neuropsicológica como el trabajo de Díaz del Guante y Molina, Neurobiología del suicidio y el trabajo de Velazco-Alzaga, Epidemiología de la violencia (Gómez \& Borges, 1996).

Las variables con las que se ha relacionado el fenómeno a una escala individual son: estado civil, tipo de ocupación, estatus ocupacional, enfermedades mentales, ingreso, edad, alcoholismo y status social (Andrés, 2005; Cook, 1985; Durkheim, 1897; Hamermesh \& Soss, 1974; Junankar, 1991; Martikainen \& Valkonen,1996; Moser, Fox \& Jones, 1984; Powell, 1958; Rushing, 1968; Yamasaki, Sakai \& Shirakawa, 2005). En el caso de los estudios a una escala agregada son: crisis políticas, grado de integración de grupos sociales, crisis económicas, desempleo, nivel de ingresos, homicidio, clima, latitud, longitud y alcoholismo (Baller \& Richardson, 2002; Baller et al., 2005; Durkheim, 1897; Wasserman \& Stack, 1995).

En la literatura podemos encontrar documentos que abordan de manera general el debate respecto al desempleo y sus efectos en la salud; la relación entre el desempleo y la mortalidad; la relación entre desempleo, ingreso y el suicidio; y por último la relación entre el tipo de ocupación, el status y el suicidio (Andrés, 2005; Cook, 1985; Hamermesh \& Soss, 1974; Junankar, 1991; Martikainen \& Valkonen, 1996; Moser, Fox \& Jones, 1984; Powell, 1958; Rushing, 1968; Yamasaki, Sakai \& Shirakawa, 2005). Dentro de las perspectivas más abordadas destacan aquellas que examinan la relación entre el desempleo y el suicidio.

En particular, Platt (1984) realiza una revisión de la literatura que aborda la relación entre el comportamiento suicida y el desempleo, que puede ser resumida como sigue: los estudios transversales a escala individual encuentran que las tasas de suicidios entre desempleados siempre son considerablemente más altas que entre los empleados. A una escala agregada no existen evidencias de una consistente relación entre desempleo y suicidio, mientras que los estudios del tipo longitudinal a escala individual apuntan a un mayor desempleo, inestabilidad en el empleo y problemas ocupacionales entre los suicidas en comparación con los no suicidas. En diversos estudios interesados en la distribución espacial de las tasas de suicidio se encuentra la presencia de agrupamientos en el espacio de tasas similares, sin embargo, la forma en que dichos agrupamientos han sido explicados difieren en el sentido de que para algunos los clústeres o agrupamientos son respuesta a un proceso de difusión mientras que para otros responden a características propias de las áreas geográficas consideradas. La forma en que los diversos estudios proceden es presentar un modelo que les permita aceptar o descartar dicho proceso de difusión. Además, se busca encontrar dentro de las variables explicativas cuáles explican mayormente la distribución de las tasas de suicidio. Así encontramos que la variable que sobresale en relación al comportamiento espacial del suicidio es la integración social (estabilidad marital, estabilidad residencial e integración religiosa).

Un estudio que para los fines de este documento es sumamente valioso es el trabajo de Manzo (2005) "El suicidio desde una perspectiva socioeconómica cultural" que pone de manifiesto la importancia de las condiciones laborales de México respecto al suicidio y en términos generales de la calidad de vida que in- 
fluyen en el comportamiento suicida. La autora menciona que el factor económico puede determinar el bienestar individual y social respecto a la satisfacción de necesidades. En el contexto mexicano (con empleos cada vez de menor calidad) es posible pensar que el factor económico puede estar influyendo en la conducta suicida, ya que al no permitir un estado de bienestar completo, el "estrés económico" rompe con la estabilidad emocional tanto individual como familiar (Manzo, 2005). Manzo (2005, 157) menciona que "habría que precisar un estado de bienestar que signifique la plena calidad de vida de la población, vista como la conciencia de satisfacción personal por medio de un empleo digno que cubra las respectivas necesidades (económicas, políticas, sociales, psicológicas, espirituales, culturales), un hecho que daría lugar a dejar al suicidio como acto poco recurrente”. El trabajo aquí mencionado parece ser de los pocos que tiene tal visión y por tanto es necesario contar con estudios actuales que retomen la preocupación de este problema y que exijan un reclamo de las condiciones económicas y laborales del país que deterioran la valoración de la vida.

En suma, podemos decir que el suicidio está relacionado con la calidad de vida de la población, en el sentido de que un incremento de suicidios en una región puede estar reflejando condiciones de vida menos satisfactorias en dicho contexto, de tal manera que los individuos toman la decisión de quitarse la vida. Además considérese que el suicidio es una respuesta extrema, por tanto las cifras del suicidio reflejan únicamente casos sociales extremos. Con esto, queremos poner énfasis en que las cifras sociales del suicidio podrían estar representando una medida del deterioro de las condiciones económicas y laborales que enfrenta la población.

\section{Aproximación al tema desde la perspectiva espacial}

Según el análisis de Durkheim, las tasas de suicidio que se agrupan en el espacio responden a patrones geográficos de integración social y de regulación, es decir, altas tasas de suicidio que se agrupan en el espacio geográfico únicamente se debe a un clúster en el mismo espacio de una débil integración social y de regulación (Baller \& Richardson, 2002, 873).

Sin embargo, en la literatura encontramos una postura que diverge de la postura de Durkheim respecto a que la débil integración social y de regulación sea la única razón de un agrupamiento de los suicidios. Tarde (1903) menciona que muchos de los comportamientos y creencias son adoptadas a través de la imitación (Baller \& Richardson, 2002, 873). Entonces si el suicidio es un proceso de imitación esto se traducirá en patrones geográficos del fenómeno (Baller \& Richardson, 2002, 874). Sin embargo, en el contexto mexicano no es válido pensar en un proceso de imitación que explique el agrupamiento en el espacio de las tasas de suicidio sino que nos apoyamos en la idea del párrafo anterior donde el agrupamiento de tasas responde al comportamiento espacial de las características del municipio o características compartidas con unidades vecinas.

La bibliografía se muestra contradictoria en este debate de si el suicidio puede deberse a un proceso de imitación, sin embargo parece ser que Durkheim tienen más partidarios. Wasserman \& Stack (1995) analizaron tasas de suicidio en Estados Unidos, concluyendo que dichas tasas no se agrupan en el espacio. Los resultados de Baller \& Richardson (2002, 886) en su documento "Integración social, imitación y patrones geográficos del suicidio" utilizando las técnicas de análisis espacial, concluyen que tanto la integración social como la imitación afectan a los patrones geográficos del suicidio. Las tasas de suicidio en Francia (1872-1876) para los departamentos del norte y sur de este país se agrupan geográficamente aún después de controlar las dimensiones múltiples de la integración social y concluyen que el efecto de imitación es más fuerte donde la integración social es mayor. Para los condados del oeste de Estados Unidos en 1990 soportan el punto de vista de Durkheim donde el agrupamiento en el espacio de las tasas de suicidio responde únicamente a las medidas de integración y las variables control. ${ }^{11}$

11 Medidas de integración social: estabilidad residencial, estabilidad marital, asistencia a una iglesia, etc. Como variables control consideraron el ingreso medio de la familia, porcentaje de desempleo, porcentaje de negros, porcentaje de nativos americanos, densidad poblacional. 
La postura de Dorling \& Gunnell (2003) aluden a que el cambio geográfico de la desesperanza puede ser explicado ampliamente como el producto del cambio geográfico de lo económico, social y demográfico. Además, diferentes estudios encuentran que áreas caracterizadas por baja integración social (midiendo la fragmentación social como una alta proporción de hogares unipersonales, alta proporción de personas divorciadas y una alta movilidad en el área) tienen las tasas más altas de suicidio (Dorling \& Gunnell, 2003, 444).

Volviendo a la discusión entre Durkheim y Tarde es importante mencionar que el primer autor no pretende decir que nunca exista la imitación pero si existe ésta no influirá en la cifra social, es decir que los efectos de la imitación no son apreciables a través de las cifras de la estadística. Durkheim (1897) predice que la incidencia del suicidio en algunas regiones del país está determinada en gran medida por un clúster de débil integración social y de regulación en estas regiones. Entonces estos factores (de la integración social y de regulación) explicarán las regiones de altas tasas de suicidio en la medida que éstos se agrupen en el espacio y sean predictores poderosos del suicidio.

Baller \& Richardson (2002) mencionan que una insuficiente integración social procrea individualismo y suicidio del tipo egoísta. ${ }^{12}$ El suicidio por razones de anomia económica, muy vinculado con nuestro tema de interés, el materialismo económico (los apetitos han sido liberados por toda autoridad que los limite) es el medio en el que el hombre se encuentra en un estado de eterno descontento (aspiraciones más altas), donde al menor revés que le sobrevenga le faltan las fuerzas para soportarlo (Durkheim, 1897).

Finalmente, el trabajo de Dorling \& Gunnell (2003, 875) para Gran Bretaña en el periodo de 1980 a 2000 considera tres componentes claves de la integración social: la migración (proporción de migran- tes internos), proporción de personas que no se encuentran trabajando y proporción de solteros. Estos autores encuentran que el efecto de la migración sobre el suicidio impacta más en edades mayores a los 45 años y el hecho de no trabajar o estar desempleado afecta en mayor proporción a los jóvenes menores a 45 años, por lo que en este trabajo se sospecha que el aumento del suicidio en edades jóvenes sea una respuesta a la falta de oportunidades en el mercado laboral y en el sistema educativo. Baller \& Richardson $(2002,444)$ consideran la estabilidad residencial (migración), la estabilidad marital (divorcio) y la integración religiosa e incluyen como variables control en su modelo el ingreso medio familiar y el porcentaje de la fuerza laboral que se encuentra empleada.

En consecuencia, desde una aproximación espacial al fenómeno no parece haber evidencia consistente de la relevancia del mercado laboral en la incidencia del suicidio pues se encuentra más relacionada con la integración social. Sin embargo, la tarea de este documento es la de indagar y comprobar si el planteamiento condiciones laborales-suicido es válida. Esta revisión fue de gran utilidad en la construcción del modelo de regresión espacial en el apartado 6.

\section{Variables del mercado laboral}

Ahora bien, ¿hay razones para suponer que el mercado laboral tiene alguna relación con la incidencia del suicidio? "El trabajo es una parte primordial de la vida, por el tiempo que nos ocupa y porque es un pilar de la integración social y autoestima de las personas" (Anker, Chernyshev, Egger, Meharan \& Ritter, 2003, 161). Por tanto, siendo el trabajo una parte tan esencial en el ser humano y en un contexto de condiciones laborales que cada vez son más precarias consideramos válido plantearse tal cuestionamiento. Al poner atención en las condiciones del mercado laboral, en la literatura consultada se pone mayor énfasis al desempleo, sin embargo, el problema de desempleo en los países de dichos

12 Los tres tipos de suicidio que define Durkheim son: (I) El suicidio egoísta que procede cuando los hombres no perciben ya la razón de estar en la vida; (II) El suicidio altruista, donde la razón les parece estar fuera de la misma vida y (III) El suicidio anómico donde la actividad del hombre está desorganizada y por esta razón sufre. 
estudios representa una problemática totalmente diferente al desempleo en México. Así mientras la variable de desempleo resulta ser una variable significativa en otros países como Estados Unidos, Gran Bretaña, etc. en el caso mexicano no es así, y el desempleo no es un indicador que abarque el conjunto de problemas del mercado de trabajo (García, $1999,10)$, por lo que nuestro marco de referencia debe ser ampliado y tomar indicadores que reflejen de mejor manera las condiciones del mercado laboral. No queremos pretender insinuar que las malas condiciones laborales o precarias son la explicación completa de la incidencia de los suicidios en nuestro país pero si es de nuestro interés el plantear la hipótesis de que las condiciones laborales tienen algún efecto en la cifra social de los suicidios.

La idea de relacionar los suicidios con las condiciones económicas es soportada por diversos estudios que han intentado encontrar una relación entre diversas variables de la dimensión económica y el suicidio. Para fines de reflexión quisiéramos indagar sobre la importancia de que la población posea empleos de calidad y la pertinencia de algunos indicadores del ámbito laboral que reflejen los niveles de vida de la población mexicana, por lo que en los siguientes párrafos se hace un esfuerzo por examinar los conceptos de trabajo decente y precariedad.

Antes de intentar definir al trabajo decente, debemos estar conscientes que en el análisis del mercado laboral existe multiplicidad de conceptos y a la vez las recomendaciones internacionales respecto a indicadores presentan también esta multiplicidad. No hay consenso, por tanto, en la forma de identificar las carencias ocupacionales, esto debido a las diferencias en la concepción de los orígenes de tales carencias y a la transformación en el tiempo según las estrategias económicas y de desarrollo tomadas (García, 2004).

Así, García (2007) presenta la evolución conceptual de la situación laboral, comenzando por los conceptos de desempleo, subempleo e informalidad y temas más recientes como flexibilidad, exclusión social o vulnerabilidad económica y social. En este recorrido encontramos conceptos como el de precariedad y trabajo decente que resultan de gran interés para el propósito de nuestro trabajo.
En el caso de precariedad como se mencionó no existe una definición exclusiva, sin embargo, si existen puntos de convergencia. Por tanto podemos decir que "precariedad" refiere a inestabilidad en el empleo, empleos sin protección social y vulnerabilidad social y económica (García, 2007). Por otro lado, el concepto de trabajo decente considera todas las clases de trabajo que se desenvuelve en condiciones de libertad, equidad, seguridad y dignidad humana (Ghai, 2003). El trabajo decente "puede referirse a la existencia de oportunidades de empleo; trabajos que no sean peligrosos o forzosos, remuneración suficiente; jornada laboral que no sea atípica o extemporánea; estabilidad y seguridad; formas para conciliar el trabajo y la vida familiar; trato no discriminado; protección social; diálogo social por medio de los sindicatos o de la negociación colectiva; entorno socioeconómico propicio al trabajo decente" (García, 2007). En términos generales, el concepto de trabajo decente busca conocer con mayor profundidad las dimensiones para hacer diferenciación entre buenos y malos empleos (García, 2004). Hemos puesto aquí mayor énfasis en el trabajo decente y la precariedad, esto lo hacemos con el único fin de llegar a la conclusión de que en términos generales las ocupaciones de calidad, no precarias, dignas o decentes, como menciona Oliveira, tienen en común abarcar varias dimensiones que debieran cumplirse en el ideal, estas dimensiones son: la económica, que implica la disponibilidad de empleos e ingresos adecuados; la normativa, que se refiere a la promoción de los derechos del trabajador; y la de seguridad laboral, relativa a la protección social al trabajador (Oliveira, 2006). Diversos autores afirman que el acceso a un empleo de calidad es un derecho y que el aprovechamiento adecuado de la mano de obra depende no sólo del monto de empleos generados sino también de la calidad de las ocupaciones (Giorguli, 2005; Alba et al., 2006).

La política de empleo se ha orientado más hacia los mecanismos financieros y económicos en la promesa de generación de empleos, lo cual evidentemente no se ha logrado. Por tanto, la propuesta es poner atención sobre aquellos sectores que están generando empleos decentes (García, 2009). Parece ser que el crecimiento económico es la meta que supone aliviara las condiciones de vida de la población, sin 
embargo, puede darse el crecimiento económico mientras la población sigue deprimida en trabajos precarios e inaceptables. Se insiste por tanto, en la importancia de generar crecimiento económico con generación de empleos decentes (García, 2009) y una distribución de la riqueza más equitativa.

El trabajo decente no sólo es importante en un nivel agregado sino que es relevante en términos de la calidad de vida de los individuos. Bajo el esquema que plantea Amartya Sen (1999) en su concepción de desarrollo como expansión de las libertades menciona algunos puntos en los que el individuo es privado de sus derechos económicos cuando la distribución de la riqueza se da de manera desigual. En esta perspectiva los trabajos de mala calidad estimulan una limitación a las libertades de los individuos, según Sen (1999):

- Privación de los derechos económicos a los individuos. Los individuos deberían tener la oportunidad de consumir, producir o realizar intercambios.

- Privación de las oportunidades sociales que no le permiten participar eficazmente en las actividades económicas y políticas.

- Seguridad protectora que proporcione una red de protección social que impida a la población caer en miseria, inanición o muerte.

El trabajo decente es afín a los propósitos del desarrollo desde la perspectiva del Desarrollo Humano. Éste consiste en la expansión de las capacidades y oportunidades de las personas para vivir una vida digna, productiva y creativa. Ubica a la persona en el centro del desarrollo, por lo que establece que el desarrollo es de, para y por las personas. Las capacidades básicas, pero no únicas, del Desarrollo Humano, son la capacidad de vivir una vida digna y saludable, de adquirir conocimientos y habilidades, y de contar con los ingresos suficientes para una vida digna (OIT, 2009).

Una vez que hemos presentado las aproximaciones al tema de interés desde la perspectiva espacial y la de mercados de trabajo, el siguiente punto será definir la metodología y los objetivos de este trabajo.

\section{Metodología y fuentes de información}

En este documento buscamos responder a la pregunta de si existe una relación positiva entre la incidencia del suicidio y malas condiciones laborales. Para esto será necesario examinar cual es la distribución espacial en nuestro país de las tasas de suicidios en el periodo 2000-2004 y si hay presencia de clústeres en el espacio de las tasas de suicidio para este periodo.

\subsection{Fuentes disponibles}

Para estudiar en México el fenómeno de los suicidios contamos con dos fuentes: los registros administrativos de Estadísticas Vitales y las Estadísticas de Intentos de Suicidio y Suicidios (EISS). La primera fuente (cifra demográfica) contiene información proporcionada por las estadísticas de defunciones bajo las causas de muerte accidentales o violentas originadas por lesiones autoinfligidas intencionalmente y la segunda fuente (cifra legal) refiere a los certificados por el Ministerio Público cuya información se recaba a través de las Estadísticas de Intentos de Suicidio y Suicidios. Al comparar dichas cifras encontramos importantes diferencias. Este diferencial entre ambas fuentes puede deberse a: (a) Casos en los que la muerte registrada en el certificado de defunción corresponde a lesiones autoinflingidas intencionalmente y estos no llegan a conocimiento del Ministerio Público; (b) casos en los que el ministerio público determina que el deceso no corresponde a un suicidio; (c) posibles rezagos en el manejo de información entre el Instituto y las fuentes informantes del Ministerio Público. Cuando los registros del ministerio público son mayores a las defunciones representa a suicidios declarados como tales por el Ministerio Público pero que no se cuenta con un certificado de defunción.

Estas diferencias son una evidencia de la calidad deficiente en las fuentes con que contamos. Así, los resultados de esta investigación pudieran verse afectados no sólo en una subestimación del nivel de incidencia del fenómeno sino que además se altere el patrón greografico del suicidio debido a la descentralización de los registros lo cual hace que la calidad de información sea diferenciada a lo largo 
del territorio del país. Esto provocará que en zonas de cobertura deficiente se pudiera suponer que el fenómeno en esa región es bajo cuando no necesariamente esto sea cierto. La cifra legal corresponde al $80 \%$ de la cifra demográfica y el comportamiento es diferenciado según la entidad, siendo Guanajuato, Puebla, Coahuila, Jalisco, Tamaulipas, Chiapas, Distrito Federal, Sinaloa, Durango, Quintana Roo, Oaxaca e Hidalgo las entidades con una mayor diferencia porcentual.

Para fines de esta trabajo se tomará la cifra demográfica que representa todos los casos en los que la causa de muerte corresponde a la claves de lesiones autoinflingidas X60 a X84 de la Décima Revisión de la Clasificación Internacional de Enfermedades (CIE 10) por ser la fuente de mejor calidad en la información respecto a la EISS. Para la obtención de las tasas utilizaremos los registros de las defunciones correspondientes a los años de 2000 al 2004 y la población recorrida al 30 de junio de 2002.

El objetivo de los siguientes párrafos es determinar los indicadores asociados a las variables que se incorporarán en el análisis. El primer punto es reflexionar sobre los indicadores propuestos del concepto de trabajo decente y por último definir los indicadores de otras variables relacionadas con el fenómeno del suicidio.

\subsection{Variables del mercado laboral}

El concepto de trabajo decente tiene facetas tanto cuantitativas como cualitativas y el problema es contar con indicadores que puedan abarcar todas estas facetas y las interrelaciones que las unen (Ghai, 2003). Diversos trabajos realizan el esfuerzo de reflexionar respecto a los indicadores que abordan las aristas del concepto del trabajo decente y proponer indicadores que permiten la medición para evaluar los progresos a lo largo del tiempo y realizar comparaciones entre países (Ghai, 2003; Anker et al., 2003; García, 2004; Bescond, Châtaignier \& Mehran, 2003). Aquí tomaremos la propuesta de Anker et al. (2003) Estos autores plantean la medición de trabajo decente con algunos indicadores estadísticos que corresponden a once categorías de medición: (i) Oportunidades de empleo, (ii) Trabajo inadmisible, (iii) Remuneración insuficiente y trabajo productivo, (iv) Jornada laboral decente, (v) Estabilidad y seguridad de empleo, (vi) Conciliación del trabajo con la vida familiar, (vii) Trato justo en el trabajo, (viii) Seguridad en el trabajo, (ix) Protección social, (x) Diálogo social y relaciones laborales, (xi) Entorno socioeconómico del trabajo decente. Con base en esta propuesta a continuación presentamos algunos indicadores que es posible obtener con la muestra censal del 2000.

- Tasa de desempleo como un indicador de la falta de oportunidades de empleo. Consideramos pertinente incluir este indicador no únicamente por el hecho que representa una medida de las personas que se encuentran en situación de desventaja y por tanto más vulnerables al evento del suicidio sino que además resulta ser un indicador que aparece reiteradamente en la literatura relacionada con el suicidio.

- Ocupados con remuneración por debajo de los dos salarios mínimos, indicador de la categoría de trabajo remunerador y trabajo productivo. Este indicador es un indicador de los más usados tanto en los estudios del suicidio como en los del mercado laboral, así que, es necesario mostrar el tipo de relación que pudiera guardar respecto a nuestro fenómeno de interés.

- Jornada laboral excesiva, indicador indirecto de jornada laboral decente. Proporción de ocupados que laboran una jornada laboral que excede las 48 horas semanales. Este indicador también es una medida de conciliación del trabajo con la vida familiar y de seguridad en el trabajo ya que jornadas laborales largas incrementa el riesgo de accidentes y enfermedades. Aunque este indicador no ha sido abordado desde la perspectiva del análisis espacial, aquí consideramos preponderante su inclusión pues una alta proporción de trabajadores con una jornada excesiva representa un mayor número de individuos en empleos demandantes. Esto a su vez pudiera implicar deterioro en la calidad de vida de los trabajadores y por tanto en mayores probabilidades de incidencia del suicidio. 
- Ocupados sin la prestación de servicios médicos como un indicador de protección social. Consideramos relevante su incorporación pues dicho indicador además de ser una arista del trabajo precario también representa una medida indirecta del grado de exclusión social que enfrentan los trabajadores al negárseles el acceso a las instituciones de bienestar social.

Estos indicadores representan algunas de las condiciones desfavorables en materia del mercado laboral, sin embargo, dichos indicadores representan las condiciones laborales que enfrentan la población que se declara como económicamente activa y esto corresponde al 50,7\% de la población total mayor a los 12 años de edad. Así que, el 49,3\% restante se declara no participante en las actividades económicas y dentro de estos rescatamos más adelante a los que declaran no trabajar por causas diferentes a la incapacidad, jubilación, ser estudiante, o dedicarse a los quehaceres del hogar (estos representan el 12\% de la población mayor a los 12 años de edad).

\subsection{Otras variables relacionadas con el suicidio}

Los indicadores que aquí se han propuesto tratan de cuantificar algunas aristas del trabajo decente, pero además es importante también tomar en cuenta el contexto económico y social de los municipios que condiciona la sostenibilidad del trabajo decente. Los indicadores que se tomarán son el grado de urbanización del municipio y el coeficiente de Gini como un indicador de desigualdad de ingresos. El primero representa un indicador del grado de desarrollo, servicios disponibles y el tipo de empleos a disposición de la población. El índice de Gini es, por tanto, una medida de la privación relativa de la población en el municipio, la población se encuentra excluida de los beneficios (riqueza) que otro grupo de la población en el mismo municipio puede disfrutar.

En este documento tomaremos como un acercamiento al concepto de integración social el porcentaje de hogares unipersonales y el porcentaje de población que ni trabaja ni estudia; este último también representa un indicador del mercado labo- ral en un sentido indirecto, dado que no refleja las condiciones laborales imperantes sino el porcentaje de población excluida de participar en el mercado laboral y por tanto excluidas de derechos económicos y sociales.

Ahora bien, la edad y el sexo son dos variables trascendentales en la disciplina de la demografía pues estas nos permiten identificar procesos diferenciados. En nuestro caso no pondremos atención en el fenómeno del suicidio según edad y sexo ya que siendo el suicidio un evento raro, el grado de desagregación que se requeriría no es adecuado para la metodología que usaremos. Así que tomaremos las tasas de suicidio en general controlando por la estructura por edad de la población. Además controlaremos por los indicadores demográficos: razón hombre-mujer y la edad media del municipio.

Por otra parte, debido a que en otros estudios el homicidio y el alcoholismo son factores asociados con el fenómeno del suicido en la tesis serán consideradas como variables control. Para esto, tomaremos como indicadores la tasa de homicidio y la tasa de muerte por cirrosis. Además, introduciremos la intensidad migratoria a los Estados Unidos como variable control, pues como habíamos visto en la bibliografía, áreas caracterizadas por una alta movilidad son regiones de altas tasas de suicidio (Dorling \& Gunnell, 2003). El siguiente punto es presentar el procedimiento en el análisis y una breve explicación de los instrumentos metodológicos en el análisis espacial.

\section{Análisis espacial}

El análisis espacial está comprendido en dos pasos: Visualización y exploración, Modelo de Regresión espacial. Dicha metodología nos llevará a contestar si existe una relación positiva entre tasas de suicidio y malas condiciones laborales.

\subsection{Visualización y exploración}

Hemos mencionado aquí como el espacio juega un papel relevante en el análisis del fenómeno que se desea analizar, y como primer paso se pretende explorar la distribución geográfica de las tasas de inci- 
dencia y verificar si existe auto-correlación ${ }^{13}$ espacial que pudiera generar problemas en las estimaciones de un modelo de regresión lineal. La auto-correlación espacial se refiere a la correlación de la variable con ella misma a través del espacio. Para comprobar si existen agrupamientos de tasas en el espacio puede ser evaluada tanto global como localmente. Una medida global es el estadístico I de Moran que se refiere a la relación entre la tasa de suicidio y el promedio de las tasas de sus vecinos (pendiente en una regresión lineal entre los valores de la tasa de los municipios y el promedio de sus vecinos). Así, el estadístico I de Moran es una medida resumen de la auto-correlación de todo el territorio, si el valor de I de Moran es +1 representa una fuerte autocorrelación positiva, si es -1 representa una fuerte auto-correlación negativa y una I de Moran cercana a cero representa la ausencia de asociación espacial. Para esto es necesario definir las áreas que consideraremos cercanas o vecinas entre sí en una matriz de pesos geográficos. En este trabajo se tomará una matriz de continuidad de reina ${ }^{14}$ que considera como vecinos a quienes comparten bordes y vértices. El cálculo del estadístico I de Moran será obtenido con el software "OpenGeoda"15. La expresión formal del estadístico es:

$$
\mathrm{I}=\frac{\sum_{\mathrm{i}} \sum_{\mathrm{j}} \mathrm{w}_{\mathrm{ij}}\left(\mathrm{Y}_{\mathrm{i}}-\overline{\mathrm{Y}}\right)\left(\mathrm{Y}_{\mathrm{j}}-\overline{\mathrm{Y}}\right)}{\sum_{\mathrm{i}}\left(\mathrm{Y}_{\mathrm{i}}-\overline{\mathrm{Y}}\right)^{2}}
$$

donde wij es la matriz de pesos geográficos, ${ }^{16}$ Yi es la tasa de suicidio en el municipio i, Yj es la tasa de suicidio en de los municipios vecinos al municipio $i$ y Y es la tasa promedio de suicidio de todo el país.

Para probar la existencia de clústeres localmente se usarán los indicadores de asociación espacial LISA que identifica los clústeres de valores similares, cuando el valor que asume la variable en una área geográfica es más similar a sus vecinos de lo que hubiéramos esperado bajo el supuesto de aleatorización espacial. Es decir, que el agrupamiento de tasas altas o bajas no pudo haberse generado por azar. Además identifica aquellas áreas con incidencia baja $y$ áreas vecinas con incidencia alta y viceversa. Estos indicadores tienen la ventaja de especificidad que a diferencia del estadístico I de Moran (que al ser una medida global) puede ocultar la dependencia espacial para algunas regiones.

\subsection{Modelo de regresión espacial}

Si en el punto anterior se verifica la existencia de auto-correlación espacial, el siguiente paso será establecer un modelo de regresión espacial que nos permitirá verificar si una mayor incidencia (o menor) de suicidios en los municipios está relacionado con las variables de integración social y del mercado laboral. Es así que se elegirá un modelo espacial autoregresivo ${ }^{17}$ basado en los resultados del diagnóstico de auto-correlación espacial: modelo de rezago espacial vs modelo de error. Se busca un modelo en el cual la auto-correlación entre la variable dependiente y en las unidades vecinas deje de ser significativa una vez que se controla por las características de los municipios. A continuación presentamos una breve descripción de dichos modelos: ${ }^{18}$

\subsubsection{Modelo de rezago espacial (Modelo Spacial Lag)}

Este modelo es también conocido como "modelo de contagio real", ya que se asume que el valor de

13 Si no existe autocorrelación espacial no es necesario generar un modelo con técnicas de análisis espacial pues un modelo de regresión lineal simple pudiera bastar para dar cuenta de la relación de nuestra variable dependendiente (suicidio) con las variables explicativas. Si la autocorrelación espacial es significativa implica que hay dependencia en los datos y por tanto se estaría violando el supuesto de independencia de la muestra en un modelo de regresión lineal simple.

14 Existen matrices basadas en el número de vecinos, matrices basadas en distancia y matrices de continuidad. Las matrices de continuidad sólo pueden definirse para polígonos y hay de dos tipos: Reina donde los vecinos son quienes comparten bordes y vértices y el de torre quienes comparten únicamente bordes.

15 Desarrollado por "Geoda Center for Geospatial Analysis and Computation".

16 En esta matriz se guardan las relaciones entre municipios (si son vecinos o no).

17 Los modelos autorregresivos modelan el proceso espacial de tal forma que la variable dependiente en un área geográfica depende de los valores observados en las unidades vecinas después de controlar por otras variables explicativas.

18 Para una explicación más detallada referirse a Anselin \& Bera (1998), Baller et al. (2001) y Anselin (2002). 
la variable dependiente en un área geográfica estará influenciado por los valores de la variable en sus vecinos. En este modelo el efecto espacial se incorpora como un predictor (variable lag de rezago espacial) y está expresado como sigue:

$$
y=p W y+X \beta+\varepsilon
$$

Donde:

$\mathrm{y}=$ es el vector de la variable dependiente

$\mathrm{p}=$ parámetro de la variable espacial de rezago a ser estimado

$\mathrm{W}=$ es la matriz de de pesos greograficos

$\mathrm{X}=$ es la matriz de variables independientes

$\beta=$ vector de parámetros de la regresión a ser estimados

$\varepsilon=$ es el vector de errores no correlacionados

Así, este modelo incorpora los efectos espaciales al incluir una variable de rezago como predictor adicional. El parámetro de la variable de rezago representa la dependencia espacial y mide la influencia promedio en las observaciones por las observaciones en las áreas vecinas.

\subsubsection{Modelo espacial del error}

Este modelo es también conocido como "modelo de contagio falso", ya que se asume que la correlación espacial está sólo en el término del error, entonces la dependencia espacial es asumida como "ruido". Los posibles efectos de las variables no incluidas aparecen como errores correlacionados espacialmente. En este modelo los efectos espaciales no se incorporan como un predictor pero se consideran a través del término del error. El modelo se expresa como sigue:

$$
y=X \beta+\varepsilon ; \quad \varepsilon=p W \varepsilon+\vartheta
$$

Donde:

$\mathrm{y}=$ es el vector de la variable dependiente

$\mathrm{X}=$ es la matriz de variables independientes

$\beta=$ vector de parámetros de la regresión a ser estimados

$\varepsilon=$ es el vector de los términos del error

$\mathrm{W}=$ es la matriz de de pesos greográficos

$\mathrm{p}=$ parámetro de la variable espacial de rezago a ser estimado

$\vartheta=$ es el vector de los errores no correlacionados

Así, los pasos a seguir en la segunda parte de la metodología son:

- Obtener un modelo de regresión lineal por estimación de mínimos cuadrados ordinarios (OLS)

- Verificar si existe auto-correlación espacial de los errores del modelo OLS (I de Moran de los errores). Si el estadístico I de Moran de los errores es significativo entonces hay razones para correr un modelo de regresión espacial. Se verifica además el diagnóstico de dependencia espacial ${ }^{19}$ que nos permitirá decidir si existe o no la necesidad de correr un modelo de regresión espacial y qué modelo de regresión espacial es el modelo más adecuado, si un modelo de rezago Lag o el modelo espacial del error ${ }^{20}$. El hecho de que exista auto-correlación en los errores del modelo OLS significa que los clústeres de tasas de suicidio permanecen una vez que los clústeres de las variables independientes son controlados.

- Obtener modelo de regresión espacial si existe correlación espacial de los errores del modelo OLS

- Verificar si existe auto-correlación espacial de los errores del modelo espacial (I de Moran). Si la auto-correlación espacial desaparece esto

19 Pruebas de diagnóstico en anexo.

20 Esta estrategia metodológica es usada por Bller \& Richardson (2002). En el estudio que ellos presentan, el hecho de que los clústeres permanecieran una vez que se controlara por las variables de integración social y de regulación y que los residuos del modelo fueran mejor representados por un modelo lag sostenía la hipótesis de que dichos agrupamientos se debían a un proceso de imitación; si los errores fueran mejor explicados por un modelo del error implicaba que la explicación de los clústeres viene de clústeres de variables estructurales no medidas y esto es consistente con la perspectiva de Durkheim de que el agrupamiento de tasas en el espacio es ampliamente determinado por el agrupamiento de la integración social y de regulación (Baller \& Richardson, 2002). 
significaría que los agrupamientos de las tasas han quedado explicados una vez que se ha controlado las variables control y el efecto espacial. Si la auto-correlación permanece podría significar que el modelo considerado no ha sido el adecuado.

- Generar el mapa asociado a los residuos del modelo espacial para con esto observar las regiones con predicciones por arriba o por debajo al valor real. Generar el mapa de LISA de los residuales del modelo espacial como una medida local de la auto-correlación espacial de los errores. Este ejercicio nos permitirá identificar aquellos clústeres de tasas que el modelo no lograra explicar, es decir, una prueba local a diferencia del punto anterior que mide la auto-correlación espacial de los errores globalmente.

Así, en el siguiente apartado se presenta la visualización y exploración de la distribución geográfica del suicidio.

\section{Resultados}

\subsection{Distribución espacial de los suicidios en México}

Como hemos mencionado la incidencia del suicidio en México es diferenciada a través del espacio y, en los estudios descriptivos que se han realizado, se soporta la evidencia de que las entidades con mayor incidencia son Campeche, Quintana Roo, Tabasco y Yucatán (Hijar, Rascón, Blanco, \& López, 1996, 17; Puentes, López, \& Martínez, 2004, 102).

En la Ilustración 3 se exhibe la distribución espacial del suicidio en los municipios de la República Mexicana. Aquí puede apreciarse cierta concentración de tasas altas en la zona sureste del país (Tabasco, Campeche, Quintana Roo); otro agrupamiento en Yucatán y otro agrupamiento en la región Noroeste que abarca entidades como Baja California Sur, Sonora, Chihuahua y Coahuila. En esta última región se encuentran inmersos municipios de baja in- cidencia por lo que pudiera haber más de un agrupamiento; uno que corresponde a municipios que se encuentran en las fronteras de las entidades de Sonora, Chihuahua y Durango; el otro corresponde a municipios ubicados en la periferia del Golfo de California (Mar de Cortés) y Estados Unidos. Para regiones como la Sur Pacífico (Oaxaca, Chiapas y Guerrero), en términos generales la incidencia es muy baja pero hay algunos municipios que presentan tasas mayores a 62 suicidios por millón de habitantes pero éstos se encuentran dispersos. Además, parece haber otros agrupamientos menos evidentes en San Luis Potosí y el norte de Veracruz; otro pequeño agrupamiento que se encuentra entre Zacatecas, Nayarit y Jalisco. En Jalisco se presentan diversos municipios con tasas altas, sin embargo parecen no agruparse en el espacio. En Coahuila también se presentan tasas altas en sus municipios ubicados en la frontera con Estados Unidos de América y en la frontera con Zacatecas. Por otra parte, una gran mayoría de municipios con tasa cero se encuentran en la región Sureste del país (Oaxaca, Chiapas y Guerrero).

Esta distribución del suicidio pudiera suponer situaciones similares en contextos cercanos. Por tanto, es importante determinar si existe dependencia espacial en nuestros datos por las siguientes razones: a) la existencia de clústeres viola el supuesto de independencia de las observaciones en modelos de regresión lineal; b) la existencia de clústeres puede ser un proceso de imitación del fenómeno o consecuencia de fuerzas económicas, sociales y políticas (Sánchez, 2006, 3).

En el Anexo ilustración 4 Cluster Map ${ }^{21}$ muestra el centro de los agrupamientos espaciales de valores similares estadísticamente. Es posible apreciar asociaciones de baja incidencia ubicadas en el sur de nuestro país en Chiapas y Oaxaca. Se aprecia una importante asociación de municipios con alta incidencia en Tabasco y Campeche, otras en Yucatán, en municipios de Chihuahua y Sonora y otros en Coahuila y Nuevo León.

21 El nivel de significancia usado es 0.05. Los mapas de clústeres presentados aquí usan este mismo nivel de significancia. 
El siguiente indicador que obtenemos es el estadístico I de Moran que corresponde a 0,1739 para las tasas de suicidio de 2000 a 2004. ${ }^{22}$ El valor de la I de Moran es significativo al 99,9\%. Este indicador representa relativamente baja auto-correlación espacial positiva (Anexo Ilustración 5). Sin embargo, no olvidemos que este estadístico es una medida global y por tanto esconde comportamientos regionales. Los indicadores de asociación espacial LISA al ser medidas locales nos han permitido evidenciar la presencia de heterogeneidad espacial de las tasas de suicidio y además comprobar con rigor estadístico la existencia de clústeres.

\subsection{Modelo de regresión lineal por estimación de mínimos cuadrados ordinarios (OLS)}

En la tabla 1 se presentan los coeficientes del modelo de regresión de Mínimos Cuadrados Ordinarios. ${ }^{23}$ En términos generales, los resultados del modelo OLS apuntan a una relación negativa entre las condiciones del mercado laboral y la incidencia del suicidio. ${ }^{24}$ El efecto mayor sobre la tasa de suicidio de las condiciones laborales corresponde a la proporción de ocupados con ingresos insuficientes, teniendo un efecto multiplicativo de $0,91^{25}$. Así, un cambio en una unidad del porcentaje de población que ni trabaja ni estudia y la proporción de ocupados sin servicios médicos disminuye la tasa de suicidio del municipio en 6 y $3 \%$ respectivamente. Las variables de desempleo e intensidad migratoria re- sultan ser no significativas en el modelo. La relación entre suicidio y hogares unipersonales resulta ser negativa, contrario a lo que habíamos encontrado en la revisión bibliográfica. El efecto de cambio en una unidad porcentual de este indicador es de $24 \%$.

La variable de urbanización es altamente significativa y es la que tiene los mayores efectos sobre la tasa de incidencia. Después de esta variable le sigue la razón hombre-mujer que también es altamente significativa aunque con un efecto menor sobre la tasa de incidencia. Así, municipios con un mayor número de hombres respecto al de mujeres implica un incremento en la tasa de suicidio, un cambio en una unidad de la razón hombre-mujer incrementa la tasa en un 13\%. El nivel de incidencia de suicidio está fuertemente relacionado con el grado de urbanización del municipio. Según el modelo tenemos que la tasa de suicidio en un municipio que tiene entre el 33 y $99 \%$ de su población rural ${ }^{26}$ es $108,72 \%$ veces la tasa de un municipio con toda su población rural. ${ }^{27}$ Bajo el modelo OLS, la tasa de municipios con menos de $33 \%$ de población rural es $28,4 \%$ mayor respecto a los municipios rurales y la tasa de suicidio de un municipio que pertenece a una metrópoli es $24,57 \%$ mayor respecto a la tasa de un municipio 100\% rural. Con esto, parece haber evidencia de que los municipios con mayor riesgo al suicidio corresponden a aquellos que entre el 33 y el 99\% de su población es rural y el menor riesgo es para los municipios $100 \%$ rurales.

22 El hecho de tomar las tasas de suicidio en el periodo de 2000 a 2004 es una estrategia metodológica que otros autores realizan para asegurar la existencia de muertes. Dorling y Gunell toman periodos de diez años para asegurar la ocurrencia de muertes en cada grupo poblacional y en cada lugar que los autores analizaron (Dorling \& Gunell, 2003, 444).

23 La variable dependiente en el modelo de regresión lineal por estimación de mínimos cuadrados ordinarios y los modelos espaciales es el logaritmo natural de la tasa anualizada de 2000-2004, esto con el objeto de lograr normalidad en la variable dependiente. La ecuación del modelo OLS queda expresado de la siguiente manera:

lntasa $=3,76-0,02$ Desempleo -0,06 No trabaja - 0,03 Más 48 horas - 0,10 Ing insuf - 0,27 Unipersonal + 0,15 inte migratoria $+4,70$ rural $3399+3,38$ ruralmenos $33+3,20$ Metropoli - 2,31 Gini + 0,12 razón_hombre

24 Las pruebas de asociación locales bivariados de LISA, es decir, la asociación espacial entre el suicidio y las variables explicativas apuntan a una relación negativa entre las condiciones del mercado laboral y la incidencia del suicidio.

$25 \exp (-0,00964)=0,917$

26 Se toma aquí el término convencional de considerar como población rural aquella que vive en localidades de menos de 2500 habitantes.

27 Si suponemos un municipio con el 100\% de su población rural y el resto de indicadores es cero la tasa de suicidio de ese municipio correspondería según el modelo OLS a 0,02 (=exp(-3,76), término constante). Si el municipio no fuera $100 \%$ rural sino que entre el 33 y 99\% de su población fuera rural y el resto de indicadores es cero su tasa sería de 0,02*109,72=2.57. Así la tasa de un municipio que tiene entre 33 y 99\% población rural es 108,72\% veces mayor que la tasa de un municipio $100 \%$ rural. 


\subsection{Auto-correlación espacial de los errores del modelo OLS y elección del modelo espacial}

Una vez que se corre el modelo de regresión lineal (OLS), el modelo conserva los problemas de auto-correlación que presentaba el fenómeno del suicidio en un principio (Estadístico I de Moran de los residuales es igual a 0,1162 , en Ilustración 6). La dependencia espacial pueden ser de dos tipos: la que es considerada como un "ruido o estorbo" y la otra es sustantivamente relevante. Como ya habíamos mencionado, el problema de auto-correlación espacial implica dependencia en las observaciones y pérdida de información, existiendo dos alternativas para enfrentar este problema, el modelo de rezago y el modelo de error. El Modelo Espacial del error considera que la dependencia espacial se encuentra en el término del error y por tanto los coeficientes del modelo no se encuentran afectados por esta dependencia. Así este modelo lo que busca es mejorar las estimaciones y obtener estadísticos no sesgados. Este modelo asumiría que la dependencia espacial de la variable de suicidio es resultado de la distribución geográfica de las variables explicativas y la auto-correlación del término error. El Modelo Espacial Lag asume una relación interactiva entra la variable independiente y la dependiente en unidades vecinas. Es decir, nuestra variable en cada unidad está determinada por el valor que asumen las unidades vecinas (Sánchez, 2006, 6). Ahora bien, según las pruebas de diagnóstico ${ }^{28}$ el modelo Lag ayuda a explicar mejor el proceso de auto-correlación espacial presente en nuestro fenómeno de estudio. Según los criterios de bondad de ajuste de Akaike (AIC), el modelo que presenta el mejor ajuste es el modelo de rezago (Lag) (Tabla 1). Además de esto, el modelo Lag es más adaptable de acuerdo al abordaje que le hemos dado al tema del suicidio donde las características compartidas entre municipios dan como resultado niveles de incidencia del suicidio similares. En modelos autoregresivos (como Lag) el propósito es encontrar como la variable dependiente se re- laciona con los valores en unidades vecinas una vez que se controla por la influencia de otras variables explicativas. Así, en el fenómeno del suicidio se busca encontrar como se relaciona la tasa de suicidio en un municipio con las tasas en sus vecinos, donde las tasas de suicidio en los vecinos vienen explicadas por el efecto de las características del municipio, es un efecto indirecto de características socioeconómicas compartidas pues en realidad el modelo mide el proceso de interacción entre tasas. En conclusión, el modelo que sirve para nuestros propósitos y que además es el más beneficiado según los diagnósticos es el modelo de Rezago Espacial.

El coeficiente espacial p en el modelo Lag es altamente significativo y tiene un efecto multiplicativo sobre la tasa de 1,34 (Tabla 1). El coeficiente p da cuenta de la interacción entre las unidades, es decir, captura el efecto que las tasas de suicidio en los municipios circundantes tienen en una unidad dada. Este parámetro suele interpretarse en términos de imitación o contagio, en tanto que representa el efecto de unidades vecinas más allá de las características observadas de cada municipio. Sin embargo, es importante recordar que la incidencia de suicidio responde en cada unidad a sus propias características y que dichas tasa inciden también sobre las de sus vecinos. Por tanto, tasas similares de suicidio responden de forma indirecta a características compartidas entre municipios vecinos.

Al comparar el modelo de regresión lineal (OLS) y el modelo de regresión lineal espacial ${ }^{29}$ (Lag) no hay un cambio drástico en la magnitud de los coeficientes ni en la dirección de los efectos excepto para la intensidad migratoria cambiando su coeficiente de 0,1464 a -0,0255, sin embargo, es una variable no significativa en ambos modelos. El índice de Gini no es significativo en el modelo espacial de rezago, es decir, una vez que se captura el efecto espacial el efecto de la variable de desigualdad de ingreso ya no aporta a la explicación de nuestra variable dependiente.

\footnotetext{
28 Pruebas de diagnóstico en anexo.

29 La comparación entre un modelo de Regresión Lineal por Mínimos Cuadrados Ordinarios y los modelos espaciales es válida y ha sido aplicada por otros autores (McLaughlin, Stokes, Smith \& Nonoyama, 2007; Irwin, 2007).
} 
Tabla 1. Coeficientes no estandarizados de la regresión de mínimos cuadrados ordinarios (OLS) y regresión espacial $(\text { Lag y error) })^{30}$

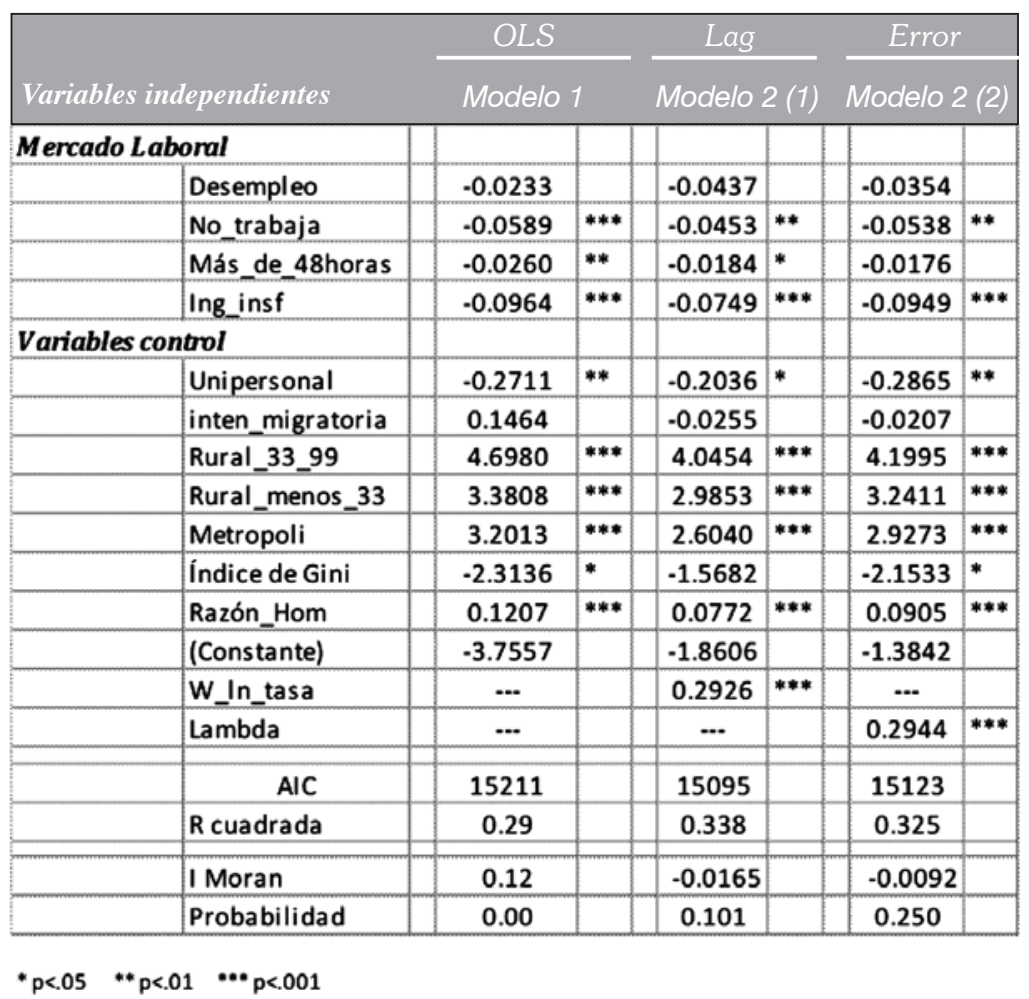

La variable desempleo no es significativa en ninguno de los modelos aquí presentados; una explicación podría ser que el desempleo es un lujo ante la falta de un seguro de desempleo. Aunque el desempleo es una variable empleada reiteradamente en la literatura como un determinante del suicidio, en el caso mexicano no existen evidencias para vincular al desempleo con el suicidio como es captado hasta este momento en otros contextos.

Al igual que en el modelo OLS, en este nuevo modelo las relaciones entre los indicadores del mercado de trabajo y el suicidio van en sentido contrario al que se esperaba. Según los resultados un incremento en el desempleo, un aumento en la proporción de población en edad de trabajar que no trabaja ni estudia, un aumento en la proporción de ocupados con jornadas laborales excesivas ó en la proporción de ocupados con ingresos insuficientes, disminuyen la incidencia del suicidio en el conjunto de los municipios de México.

Cabe mencionar que cuando tratamos de explicar un proceso de alta heterogeneidad espacial en un modelo general, los coeficientes de la regresión nacional no son capaces de modelar procesos locales como los de alta incidencia de la región del Golfo, Península de Yucatán o Norte de nuestro país. Considérese el contraste entre Baja California Sur y Oaxaca, la primera una entidad de alta incidencia y la segunda de baja incidencia, el número de municipios de cada entidad corresponde a 5 y 570

30 Fuente: Elaboración propia. 
respectivamente; los municipios de Baja California Sur tienen en promedio $14.695 \mathrm{~km}^{2}$ mientras que los de Oaxaca tienen $164 \mathrm{~km}^{2}$. Dado que en los modelos de regresión nacionales estamos atribuyendo los mismos pesos a todos los municipios del país, ${ }^{31}$ esto implica que el modelo se encuentra sesgado por regiones como la de Oaxaca. ${ }^{32}$

En el caso de las jornadas laborales por arriba a las 48 horas por semana tenemos que un incremento en el porcentaje de ocupados en esta condición disminuye la tasa de suicidio en un $1,8 \%$. La variable del mercado laboral que resulta ser más significativa es la proporción de ocupados con ingresos insuficientes y la magnitud de su coeficiente también es mayor respecto a los anteriores, un incremento en una unidad disminuye en un $7 \%$ la tasa de suicidio.

Si bien la dirección de los indicadores es contraria a lo que se esperaba, la magnitud de los coeficientes es pequeña y por tanto el efecto sobre las tasas de suicidio es casi nulo en comparación con otras variables como la urbanización del municipio. Entonces, no tenemos evidencias estadísticas para probar la hipótesis planteada inicialmente que suponía una relación positiva entre las malas condiciones laborales y el suicidio a la escala municipal. Este resultado es consistente en el modelo espacial y el modelo OLS, de hecho la dirección y magnitud de los coeficientes asociados a las variables de mercado laboral son similares en ambos modelos. Dados estos resultados será importante explorar sobre la relación entre el suicidio y otras variables.

Según el modelo espacial de rezago Lag el porcentaje de población mayor a los 12 años que no trabaja ni estudia tiene un efecto multiplicativo sobre la tasa de suicidio de 0,956, es decir, un incremento en una unidad del porcentaje de población que no trabaja ni estudia disminuye 4,4\% la tasa de suicidio.

Analizando la variable de urbanización, los resultados en el modelo sugieren que municipios con $100 \%$ de su población rural presentan las menores tasas de suicidio; le siguen los municipios que pertenecen a una metrópoli siendo sus tasas 13 veces las de los municipios rurales; luego la tasa de municipios con menos del 33\% de su población rural que es 13 veces la de los rurales y por último las tasas de municipios con un porcentaje de población rural entre 33 y 99\% es 57 veces la tasa que presentan municipios rurales. Estos resultados son sorprendentes pues el grado de urbanización no actúa de forma lineal sobre la incidencia y las mayores tasas las presentan municipios medianamente rurales.

En el modelo espacial Lag, las variables intensidad migratoria e índice de Gini ya no son estadísticamente significativas (a diferencia del modelo de regresión OLS), esto implica que si sólo atendiéramos a los resultados del modelo OLS estaríamos dando un sentido inadecuado a la influencia de estas variables al no considerar el efecto espacial.

La dirección del coeficiente de porcentaje de la población que vive sola es inversa a las tasas de suicidio, por tanto, un aumento de hogares unipersonales disminuye la tasa de suicidio. Esto va en contra de lo que predecía nuestro marco teórico, por lo que, por una parte, es necesario desarrollar teoría aplicable al contexto mexicano y, por otro lado, debemos revisar la adecuación de nuestros indicadores de integración social.

Dentro de nuestros indicadores demográficos hemos tomado la razón hombre-mujer y la edad media del municipio, sin embargo, en el modelo de elección hemos dejado fuera la última variable por razones metodológicas. En los modelos en los cuales fue incorporada la edad media, ésta resultaba ser no significativa en la explicación del fenómeno del suicidio. Aunque la razón hombre-mujer es un indicador demográfico también pudiera representar un indicador de integración social ya que una razón elevada puede ser consecuencia de alta inmigración masculina en el municipio. En este sentido la dirección del coeficiente es como se esperaba, un incremento en la razón hombre-mujer incrementa en $8 \%$ la tasa de suicidio en el municipio.

31 Cada municipio representa una observación.

32 Región donde el suicidio pudiera responder a diferentes factores. 


\subsection{Auto-correlación espacial de los errores del modelo espacial (I de Moran)}

El modelo espacial de rezago (Lag) permitió que la auto-correlación entre la variable dependiente y las unidades vecinas deje de ser significativa una vez que se controla por las características de los municipios. El valor del estadístico I de Moran de los residuales es igual a -0,0165 (Anexo Ilustración 7).

Aunque en términos generales ya no tenemos problemas de auto-correlación espacial (I de Moran es igual a -0,0165), desde una perspectiva como la de Durkheim, el agrupamiento de tasas debería desaparecer una vez que controlamos por los indicadores de integración social y nuestras variables control. Para verificar esta afirmación haremos una prueba local (LISA) de los residuos del modelo Lag para verificar si los clústeres han desaparecido.

Al observar el mapa de LISA (Anexo Ilustración 8) de los residuales del modelo Lag podemos decir que, con las variables que hemos considerado en nuestro modelo, aún contamos con municipios que pertenecen a un clúster, sin embargo, su número es reducido. Al comparar este mapa con los clústeres de suicidio iniciales vemos que el modelo ha logrado explicar los clústeres de bajas incidencias en el sur, el clúster del golfo en Tabasco y un considerable número de municipios que pertenecen a agrupamientos de tasas altas en el norte de nuestro país. Entonces, la afirmación de que los clústeres deberían desaparecer una vez que se ha controlado por las variables explicativas se cumple para una gran mayoría de municipios pero no en su totalidad. Los municipios que aún pertenecen a un clúster se encuentran ubicados en la región Noroeste del país en Sonora y Chihuahua, los cuales desde el análisis descriptivo evidenciaban un comportamiento muy especial tanto en sus tasas como en las variables explicativas. Otros se ubican en Yucatán; otros entre Nuevo León y Tamaulipas; otros entre San Luis Potosí y Guanajuato y otros entre Jalisco y Nayarit. Lo que esto significa es que para estas regiones del país el modelo no ha logrado explicar totalmente la dependencia espacial entre municipios, aunque en términos generales la dependencia espacial ya no sea un problema.
En términos generales, dados los resultados, sugerimos como una posible explicación que municipios con mejores condiciones implican mayores aspiraciones en la población y por tanto, el suicidio estaría mejor explicado no tanto por las condiciones que enfrenta la población en general como por las situaciones de desventaja para algunos miembros. Es decir, en los municipios donde la población disfruta de mejores condiciones quienes se encuentran fuera de estos beneficios pudieran considerarse en desventaja. Tal vez, como consecuencia de esto, las variables de urbanización son altamente significativas y tienen un efecto mayor sobre las tasa de suicidio.

\section{Conclusiones}

Uno de los aportes de este trabajo radica en la exploración del fenómeno a una escala no explorada que permite un mejor entendimiento del comportamiento espacial del fenómeno y reflexión sobre otros determinantes del suicidio a una escala agregada que añaden a los factores individuales ya abordados por otros estudios.

Es importante destacar la reflexión analítica sobre las posibles relaciones que se establecen entre el suicidio y diversas variables. Dentro de esta reflexión lo que debe destacarse es el esfuerzo por identificar los posibles vínculos con una arista de las condiciones económicas: el trabajo, elemento que no ha sido incorporado en los marcos teóricos relacionados con el suicidio.

El principal objetivo del trabajo fue el de explorar la relación que guarda la incidencia del suicidio y malas condiciones labores. Para este propósito fue importante evidenciar en primer lugar el comportamiento heterogéneo del fenómeno del suicidio a través del territorio mexicano utilizando las herramientas del análisis espacial y con esto mostrar que las cifras nacionales esconden comportamientos regionales importantes. Además era importante identificar la presencia de clústeres en el espacio de las tasas de suicidio para el periodo de 2000-2004, es decir, se demostró la existencia de agrupamientos en el espacio de tasas altas o bajas de suicidios. Con este objeto se presentaron medidas locales y globales de auto-correlación.

Los modelos espaciales permitieron corregir los problemas de auto-correlación espacial (es decir, la 
correlación de la variable -suicidio- con ella misma a través del espacio), con un porcentaje explicativo de variación de los datos mucho más elevado que algunos de los trabajos citados en el marco teórico. La distribución espacial de los errores nos permitió ubicar algunos agrupamientos de tasas. Esto significa que para dichas regiones del país el modelo no logro explicar la dependencia espacial en nuestra variable dependiente. En consecuencia, lo que no pudo ser explicado por el modelo nos da pautas para pensar en el ajuste de los marcos conceptuales para el suicidio en México.

La metodología aquí empleada nos permite sostener con amplio fundamento estadístico que el camino a seguir en la investigación futura será atender el tema de los comportamientos locales dado que la incidencia del suicidio parece estar respondiendo a diferentes factores según la región que se hable.

Dentro de los resultados encontrados destaca el hallazgo referente a que nuestros indicadores del mercado de trabajo no mantienen una relación positiva con la incidencia del suicidio desde la perspectiva espacial a escala municipal. En principio este hecho pudiera ser explicado por dos razones metodológicas. La primera tiene que ver con el tipo de indicadores que hemos utilizado, ya que se partió de un conjunto de indicadores que frecuentemente se utilizan en el análisis de empleos urbanos y que representan únicamente al $50,7 \%$ de la población total mayor a los 12 años, por tanto, es importante reflexionar sobre otro tipo de medidas que pudieran reflejar de mejor manera la heterogeneidad del empleo en nuestro país y la diversidad de situaciones laborales de tipo rural a escala municipal.

La segunda razón tiene que ver básicamente con las características de la información y del fenómeno mismo, dado que el suicidio es un evento "raro" estadísticamente hablando tenemos un número considerable de municipios con incidencia cero, lo cual provoca que el fenómeno del suicidio presente dos procesos claramente identificables: municipios con incidencia cero y diferencias significativas en los niveles de incidencia para municipios con tasas positivas, por tanto convendría explorar modelos que pudiesen dar cuenta de ambos procesos simultánea- mente. Una propuesta a futuro sería aplicar una técnica como la denominada "hurdle regression model" que combina un modelo binario y un modelo truncado poisson (binomial negativa) que predice el proceso de ocurrencia o no en los municipios del país y el nivel de incidencia en los municipios. Este modelo supone que son dos procesos distintos lo que está dando lugar a las dos distribuciones (cero incidencia vs. incidencia positiva) (Long \& Freese, 2002).

Además, de haber este doble proceso en el fenómeno del suicidio, al intentar aplicar un modelo general, como el que se ha presentado en esta documento, pueden estarse escondiendo procesos de carácter más específico, ya que los municipios sin incidencia de suicidio (35\% del total) pudieran estar pesado demasiado en el modelo y no permitir tener un mejor ajuste del fenómeno para ciertas regiones. Una de las sugerencias en este sentido es tomar distritos en lugar de municipios en entidades como Oaxaca, donde el número de municipios por kilómetro cuadrado es muy pequeño, o hacer una corrección por área geográfica. La primera sugerencia es apoyada en otros documentos como el de Villarreal (2002) que incorporan la perspectiva espacial en México a una escala municipal.

Dentro de nuestros indicadores del mercado de trabajo se incorporó la variable del desempleo la cual no mantiene una relación positiva con la incidencia del suicidio, lo cual es consistente con la revisión que hace Platt (1984) a una escala agregada y que refiere que no existen evidencias de una consistente relación entre desempleo y suicidio.

Algunas variables que pudieran incluirse en el modelo, con el objeto de mejorar el ajuste y conseguir una mejor explicación de la auto-correlación espacial, son: latitud/longitud y densidad poblacional, variables empleadas en otros estudios. Las dos variables de la dimensión de la integración social (el porcentaje de la población que no estudia ni trabaja y el porcentaje de hogares unipersonales) presentaron una relación negativa con la incidencia del suicidio, contrario a lo que se había encontrado en la revisión bibliográfica. Por tanto, sería deseable contar con mejores indicadores de los adherentes 
religiosos y la integración social que puedan incluirse en el modelo pues como se vio en la bibliografía, tanto la religión y la integración social tienen un vínculo destacado con el suicidio.

Desde la aproximación espacial al fenómeno, en la revisión de la literatura no parecía haber evidencia consistente de la relevancia del mercado laboral en la incidencia del suicidio. Este trabajo ha aportado evidencia estadística suficiente para sostener que de manera general no es posible afirmar que malas condiciones laborales estén provocando un incremento en la incidencia del suicidio a escala municipal, sin embargo, esta investigación abre una línea de análisis inmediata, en la cual podría no descartarse la hipótesis de una relación positiva entre malas condiciones laborales y el suicidio en regiones muy específicas.

\section{Referencias}

Alba, F et al. (2006). "El bono demográfico en los programas de las políticas públicas de México (2000-2006): un análisis introductorio". En: La situación demográfica de México, 2006. México, Consejo Nacional de Población.

Andrés, A. (2005). "Income inequality, unemployment, and suicide: a panel data analysis of 15 European countries". En: Applied Economics, 37(4):439-451.

Anker, R., Chernyshev, I., Egger, P., Meharan, F. \& Ritter, J. (2003). "La medición del trabajo decente con indicadores estadísticos". En: Revista Internacional del Trabajo, 12(2):161-195.

Anselin, L. (2002). "Under the Hood: Issues in the Specification and Interpretation of Spatial Regression Models". En: Agricultural Economics, 27( 3): 247-267.

Anselin, L. \& Bera, A. (1998). Dependence and Linear Regression models with an Introduction to Spatial Econometrics. Champaign: University of Illinois at Urbana-Champaign.

Baller, R. \& Richardson, K. (2002). "Social Integration, Imitation, and the Geographic Patterning of Suicide". En: American Sociological Review, 67(6): 873-888.

Baller, R., Shin, D. \& Richardson, K. (2005). "An extension and test of Sutherland's concept of Differential Social Organization: The geographic Clustering of Japanese Suicide and Homicide rates". En: Threatening Behavior, 35(3):343-355.

Baller, R.; Anselin, L.; Messner, S.; Deane, G. \& Hawkins, D. (2001). "Structural covariates of U.S. County homicide rates: Incorporating Spatial Effects". En: Criminology, 39(2):561-590.

Bescond, D., Châtaignier, A. \& Mehran, F. (2003). "Siete indicadores para medir el trabajo decente. Comparación internacional". En: Revista Internacional del Trabajo, 122(2): 197-231.

CONAPO, (2013). "Proyecciones de la Población 2010-2050 basadas en el XII Censo de Población y vivienda". Disponible: http://www.conapo.gob.mx/es/CONAPO/Proyecciones.

Cook, D. (1985). "A critical view of the unemployment and health debate". En: The Statistician, 34:73-82.
Dorling, D. \& Gunnell, D. (2003). "Suicide: the spatial and social components of despair in Britain 1980-2000". En: Transactions of the Institute of British Geographers, 28(4):442-460.

Durkheim, E. (1897). El suicidio. México, D.F.: Ediciones Coyoacán.

García, B. (1999), "Los problemas laborales de México a principios del siglo XXI". En: Papeles de Población, Universidad Autónoma del Estado de México, 1(21):9-19.

García, B. (2004). "La carencia de empleos satisfactorios: una discusión sobre indicadores". En: Figueroa, B. (coord.) El dato en cuestión: Un análisis de las cifras sociodemográficas, México, El Colegio de México: 461-488.

Garcia, B. (2007), "Las carencias laborales en México: Conceptos e indicadores". En: Edith Pacheco, Enrique de la Garza \& Luis Reygadas (coords.) Trabajos atípicos y precarización del empleo. México: El Colegio de México.

García, B. (2009). "Mercados de Trabajo y empleo". En: Consejo Nacional de Población (México), United Nations Population Fund y Sociedad Mexicana de Demografía, Foro Nacional "Las Políticas de Población en México. Debates y Propuestas para el Programa Nacional de Población 2008-2012". México: CONAPO.

Ghai, D. (2003). "Trabajo decente. Concepto e indicadores". En: Revista Internacional del Trabajo, 122(2):125-160.

Giorguli, S. (2005). "Demografía y economía en el México de hoy", en Consejo Nacional de Población (México), United Nations Population Fund y Sociedad Mexicana de Demografía, Foro Nacional "Las Políticas de Población en México. Debates y Propuestas para el Programa Nacional de Población 20082012". México: CONAPO

Gómez, C. \& Borges, G. (1996). "Los estudios que se han hecho en México sobre la conducta suicida:1996-1994”. En: Salud Mental, 19(1):45-55.

Hamermesh, D. \& Soss, N. (1974). "An Economic Theory of suicide". En: Journal of Political Economy, 82(1):83-98.

Hernández, H. \& Flores, R. (2011). "La evolución del suicidio en México”. En: Papeles de Población, 17(68):69-101.

Hijar M., Rascon, R., Blanco, J. \& Lopez M. (1996). "Los suicidios en México. Características sexuales y geográficas (1979-1993)". En: Salud Mental, 19(4): 14-21.

INEGI (2003). Síntesis Metodológica de las Estadísticas de Intentos de Suicidios y Suicidios. En: Instituto Nacional de Estadística, Geografía e Informática. Disponible en: www.inegi.org.mx

INEGI. Estadísticas Vitales 1990-2011. Disponible en: http:// www.inegi.org.mx/est/contenidos/Proyectos/registros/vitales/ mortalidad/default.aspx

Irwin, M. (2007). "Territories of Inequality: An essay on the Measurement and Analysis of Inequality in Grounded Place Settings". En Lobao, L.; Hooks, G. \& Tickamyer, A. (Ed.). The Sociology of Spatial Inequality, State University of New York. Estados Unidos de América.

Junankar, P. (1991). "Unemployment and mortality in England and Wales: A preliminary analysis". En: Oxford Economic Papers, 43: 305-320.

Long, J. \& Freese, J. (2002). "Regression models for categorical dependent variables using stata". Texas: StataCorp LP.

MacLaughlin, L.; Stokes, S.; Smith, J. \& Nonoyama, A. (2007). "Differential Mortality Across the United States. The influence of Place-Based Inequiality". En: Lobao, L.; Hooks, G. \& Tickamyer, A. (Ed.). The Sociology of Spatial Inequality, State University of New York. Estados Unidos de América. 
Manzo, G. (2005). "El suicidio desde una perspectiva socioeconómica cultural". En: Cuicuilco, 12(33): 153-171.

Martikainen, P. \& Valkonen T. (1996). "Excess mortality of unemployed men and women during a period of rapidly increasing unemployment". En: Lancet, 348(9032):909-12.

Moser, K., Fox, A. \& Jones, D. (1984). "Unemployment and mortality in the OPCS Longitudinal Study". En: Lancet, 2(8415):1324-1329.

OIT (2009). Informe Nacional sobre Desarrollo Humano 2011: Trabajo Decente en Paraguay. Disponible: www.undp.org. py/v3/showdocs2.aspx?d=D000133.pdf

Oliveira, O. (2006). "Jóvenes y precariedad laboral en México". En: Papeles De Población, 49:37-73.

OMS (2010). World Health Statistics Report 2010. Disponible:

http://www.who.int/gho/publications/world health_statistics/ en/index.html

Pacheco, E. (2009). Clase Mercados de Trabajo. Mimeo Maestría Demografía 2008-2010. México: El Colegio de México.

Pacheco, E. \& Flores, R. (2005). El factor trabajo en México y las metas del milenio. México, ante los desafíos de desarrollo del milenio. México, Consejo Nacional de Población.

Platt, S. (1984). "Unemployment and suicidal behavior: a review of the literature". En: Social Science y Medicine, 19(2): 93-115.
Powell, E. (1958). "Occupation, status, and suicide: Toward a redefinition of anomie". En: American Sociological Review, 23(2), 131-139.

Puentes, E., López, L. \& Martínez, T. (2004). "La mortalidad por suicidios: México 1990-2001". En: Revista Panamericana De Salud Pública, 16(2):102-109.

Rushing, W. (1968). "Income, Unemployment, and suicide: An Occupational Study", Sociological Quarterly", 9(4):493-503.

Sánchez, L (2006). Métodos para el análisis espacial. Una aplicación al estudio de la geografía de la pobreza. Guadalajara, México.

Sen, A. (1999). Development as freedom. New York, Knopf

Villarreal, A. (2002). "Political competition and violence in Mexico: Hierarchical Social Control in Local Patronage Structures". En: American Sociological Review, 67: 477-498.

Wasserman, I. \& Stack, S. (1995). "Geographic spatial autocorrelation and United States suicide patterns". En: Archives of Suicide Research, 1(2):121-129.

Yamasaki, A., Sakai, R. \& Shirakawa, T. (2005). "Low income, unemployment, and suicide mortality rates for middle-age persons in Japan”. En: Psychological Reports, 96(2):337-48. 


\section{ANEXO}

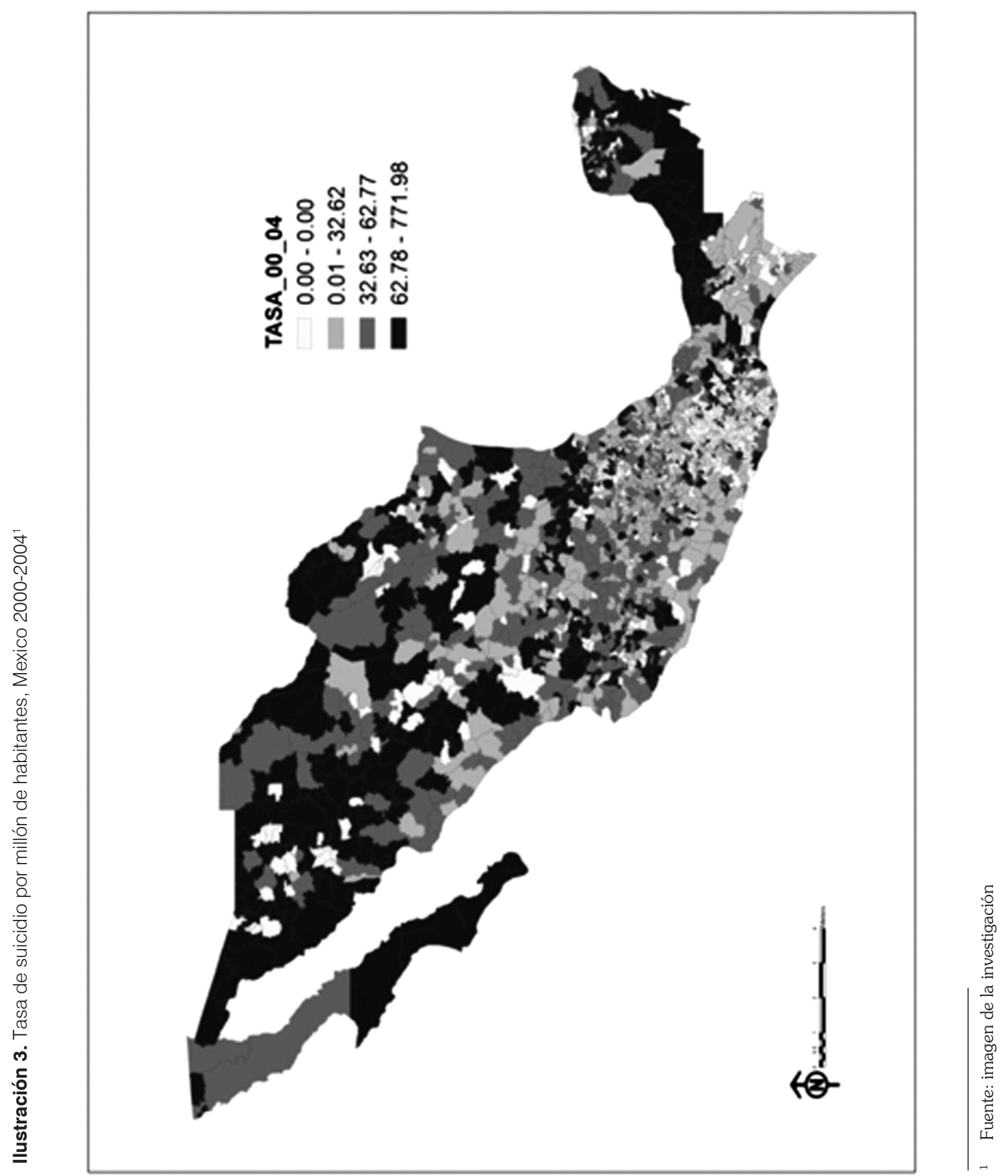




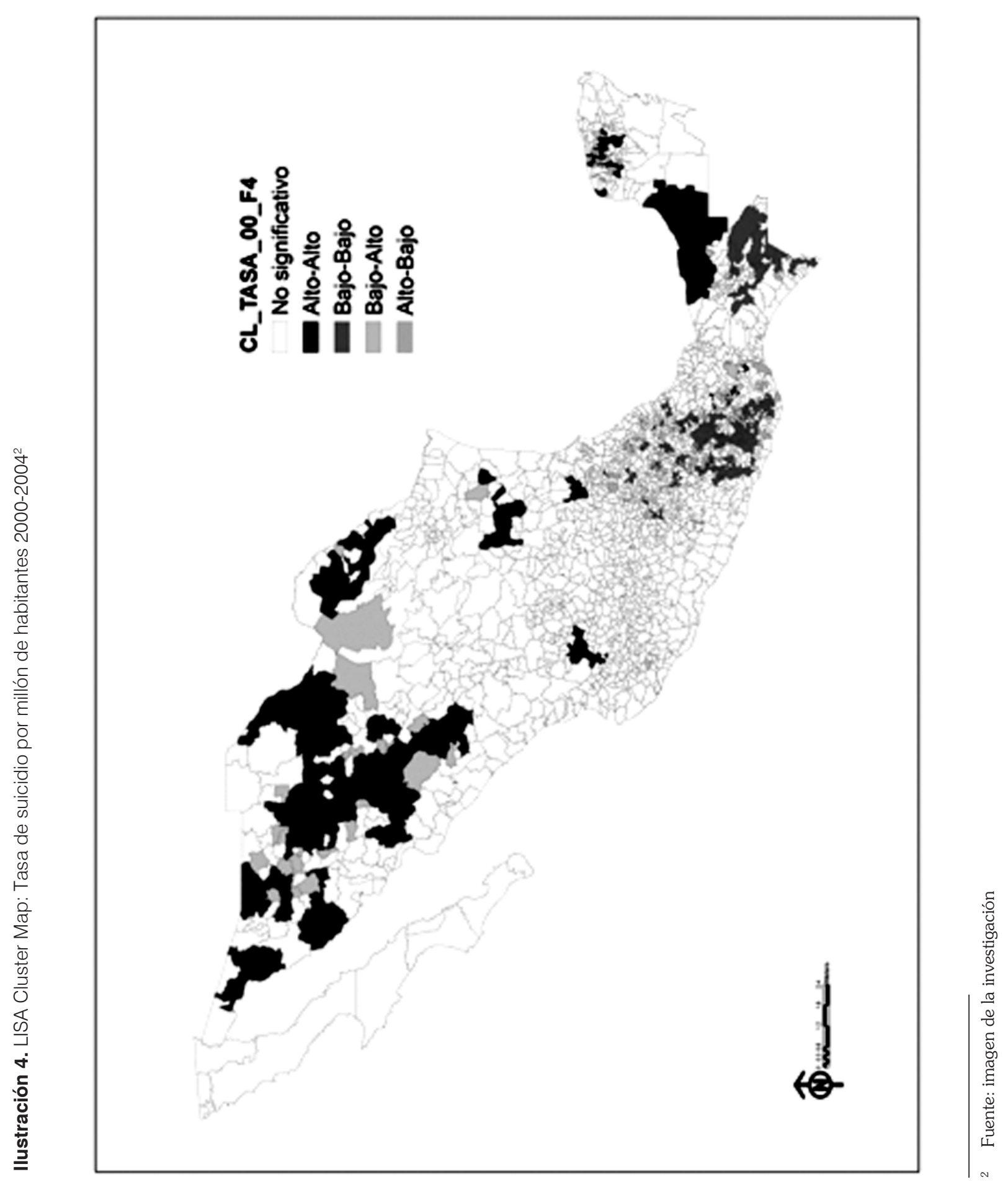


Ilustración 5. Estadístico I de Moran (tasas de suicidio). ${ }^{3}$

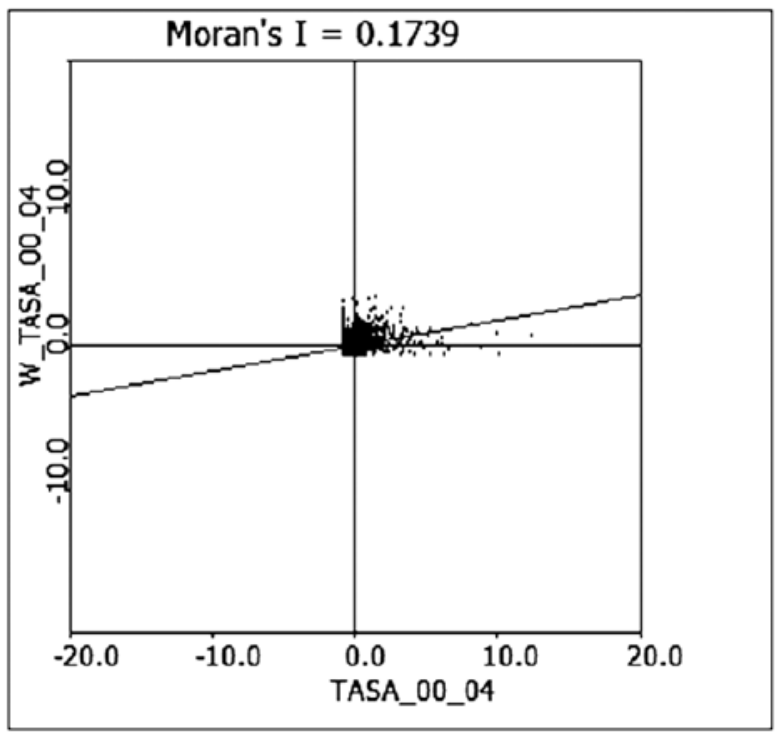

Ilustración 6. Índice I Moran y mínimos cuadrados ordinarios (OLS). ${ }^{4}$

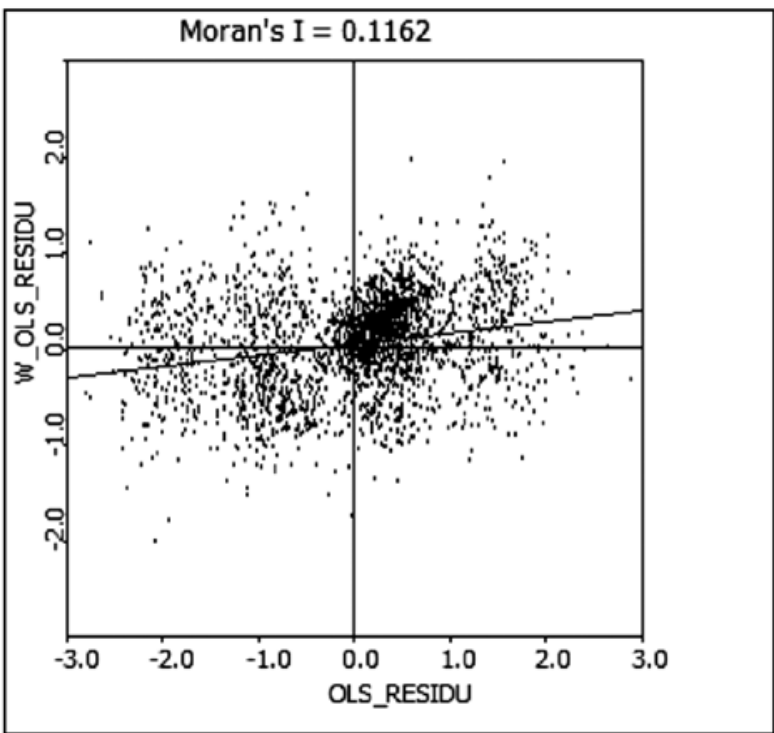

Ilustración 7. Estimación de los errores del modelo espacial (I de Moran) ${ }^{5}$

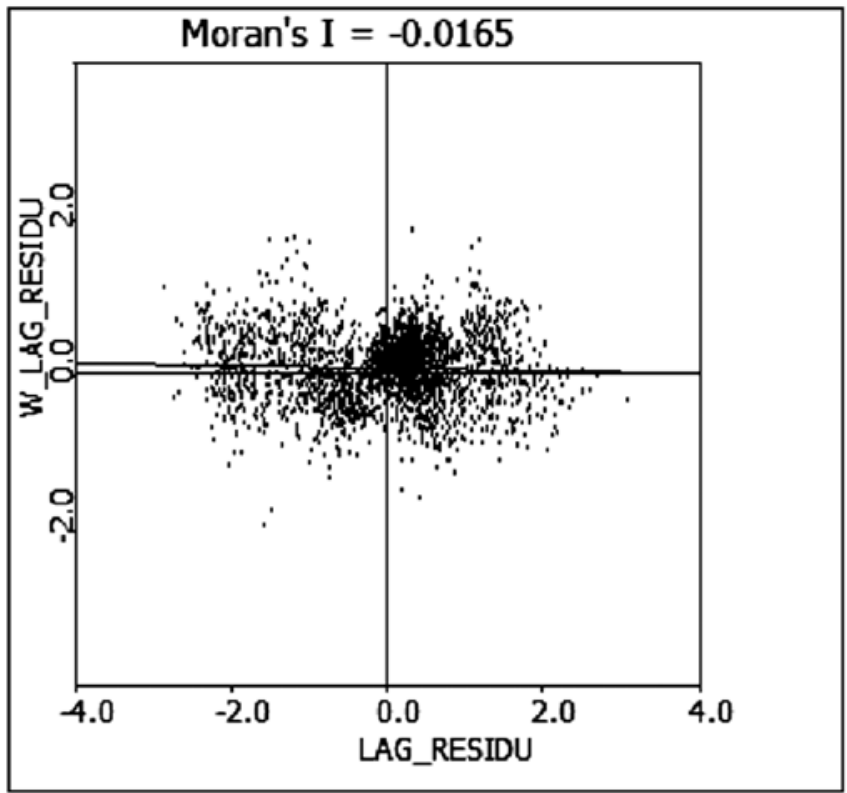

Fuente: Elaboración propia Fuente: Elaboración propia Fuente: Elaboración propia 

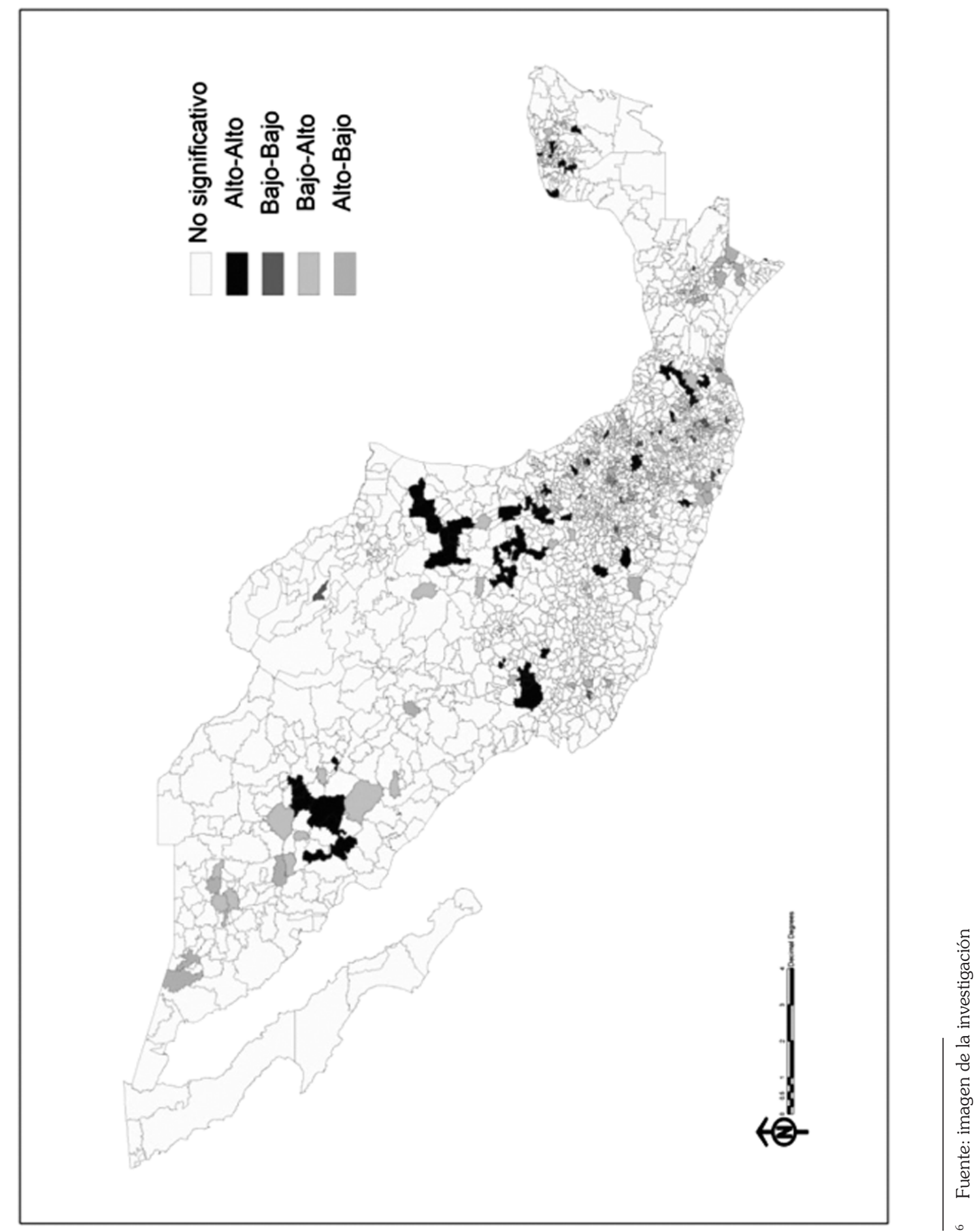

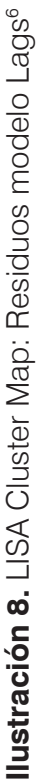




\section{Pruebas de diagnóstico}

\section{DIAGNOSTICS FOR SPATIAL DEPENDENCE}

DIAGNOSTICS FOR SPATIAL DEPENDENCE

FOR WEIGHT MATRIX : Base_regresión_queen.gal (row-standardized weights)

\section{TEST MI/DF VALUE PROB}

Moran's I (error) 0.1162129 .79217690 .0000000

Lagrange Multiplier (lag) 1132.68171900 .0000000

Robust LM (lag) 142.68897580 .0000000

Lagrange Multiplier (error) 191.80396890 .0000000

Robust LM (error) 11.81122570 .1783613

Lagrange Multiplier (SARMA) 2 134.4929447 0.0000000

La hipótesis nula en las pruebas de Lagrange Multiplier (lag) y Robust LM (Lag) es $\mathrm{p}=0$, en el modelo de rezago: $y=p W_{y}+X \beta+\varepsilon$

La hipótesis nula en las pruebas de Lagrange Multiplier (error) y Robust LM (error) es $\lambda=0$, en el modelo de error: $y=X \beta+\varepsilon ; \varepsilon=\lambda W_{\varepsilon}+\vartheta$. Así, las pruebas de diagnóstico favorecen el modelo de rezago (Lag). 